\title{
The Effect of Altered Neuronal Activity on the Development of Layers in the Lateral Geniculate Nucleus
}

\author{
V. A. Casagrande ${ }^{1,2}$ and G. J. Condo ${ }^{1}$ \\ Departments of ${ }^{1}$ Cell Biology and ${ }^{2}$ Psychology, Vanderbilt University School of Medicine, Nashville, Tennessee 37232
}

The main objective of this study was to examine the role of neural activity in the development of cell layers in the dorsal lateral geniculate nucleus (LGN). We studied this relationship in postnatal tree shrews either by completely blocking retinal ganglion cell activity with TTX or by selectively blocking activity to the developing ON-center LGN layers (1 and 2) with 2-amino-4-phosphonobutyric acid (APB), using unilateral and bilateral eye injections. All manipulations were carried out from birth (PO), when no LGN cell layers are evident, to or past the point when layers are recognizable (i.e., 1-2 weeks). Nissl-stained and cytochrome oxidase (CO)reacted material was examined for all cases.

Our results show that in the absence of activity produced by bilateral TTX injections, interlaminar spaces between cell layers do begin to develop. Retinal afferents, which are segregated at birth, remain segregated, and differential $c 0$ staining between matched sets of LGN layers is evident. The normal pace of LGN development, however, is slowed significantly: LGN cells are smaller and interlaminar spaces are narrower than are seen in age-matched controls. Unilateral TTX injections produce similar, but more dramatic and asymmetric, effects on LGN cells, perhaps because cells are at a competitive disadvantage relative to their normally innervated counterparts in cortex. Combining unilateral eye enucleation at PO with subsequent TTX treatment of the other eye clearly demonstrates that axons from the remaining eye are capable of producing their normal complement of LGN layers. The development of the LGN ON-center layers, 1 and 2 , and the interlaminar space between them are more affected by TTX treatment than are the other layers. By contrast, APB eye injections do not selectively affect the development of the ON-center layers, but do result in some slowing of overall LGN development.

Taken together, these results suggest that activity of retinal afferents is not essential for initiating interlaminar space formation, but is important for the normal pace of maturation of LGN cell layers.

\footnotetext{
Received June 20, 1986; revised July 21, 1987; accepted July 23, 1987.

We would like to thank Dr. Mark Dubin for useful discussions on the project and Drs. Jim Hutchins, Sherre Florence, and John Wall for helpful comments on the manuscript. We also gratefully acknowledge the expert technical assistance we received from Dr. Lance Durden, Dr. Hutchins, Sam Marvin, Aurora Buck, Lisa Bruno, and Julie Mavity during various phases of the study, and Vera Murphy's help in typing the manuscript. This work was supported by National Institutes of Health Grant EY05038 and Career Development Award K04-EY00223 to V.A.C. and Postdoctoral Fellowship F32-EY05770 to G.J.C.

Correspondence should be addressed to Dr. V. A. Casagrande, Department of Cell Biology, Vanderbilt University School of Medicine, Nashville, TN 37232.

Copyright (C) 1988 Society for Neuroscience $0270-6474 / 88 / 020395-22 \$ 02.00 / 0$
}

One of the central issues in developmental neurobiology concerns the differentiation of orderly arrangements of cells and connections. In some systems, the orderly mature arrangement of cells depends critically on the presence of one set of afferents for normal expression. This is the case for the lamination pattern characteristic of the dorsal lateral geniculate nucleus (LGN), which depends on the presence of afferent projections from the retina. We have been examining the developmental relationship betwecn retinal afferents and their postsynaptic targets in the LGN of the tree shrew (Tupaia belangeri), a species that is born before the formation of cell layers but after retinal afferents are segregated within the nucleus. In this species, we have demonstrated that complete removal of retinal input at birth prevents the formation of interlaminar spaces between cell layers (i.e., prevents cellular lamination as defined cytoarchitectonically), but allows the surviving cells in the nucleus to express normal differences in morphology (cytologically defined lamination) and to connect topographically with striate cortex (Brunso-Bechtold and Casagrande, 1981; Brunso-Bechtold et al., 1983). Others have extended these observations by demonstrating the same results in prenatal mink and ferrets enucleated prior to innervation of the LGN. That is, cell layers defined cytoarchitectonically fail to form, but topographic connections with cortex and some cytological features of the nucleus do develop (Guillery et al., 1985).

Our experiments have also shown that when both eyes are removed at birth, the rate of LGN cell maturation is slowed. If eyes are removed during the period of interlaminar space formation and the animal is permitted to survive to maturity, interlaminar space formation appears "frozen" at the stage of development when the eyes were removed, except that the interlaminar spaces present widen to adult levels (Brunso-Bechtold and Casagrande, 1981, 1985). All of these results suggest that, although some aspects of LGN ontogeny are either intrinsic or dependent on extraretinal afferents, afferents from the retina are critical for initiating the formation of LGN cell layers as defined cytoarchitectonically. The question, then, is, How do retinal afferent axons influence the spatial arrangement of their postsynaptic targets to produce cell layers?

Several lines of evidence have implicated neural activity as having a trophic role in shaping neural development (Changeux and Danchin, 1976; von der Marlsburg and Willshaw, 1981; Schmidt and Edwards, 1983; Stryker and Harris, 1986). If impulse activity is a cue employed by optic fibers to organize geniculate cells into layers, we reasoned that altering or turning off such activity should alter, interrupt, or perhaps prevent geniculate cell layer formation. In the present study, we tested this idca using 2 paradigms. We cxamincd the effect of completely or selectively blocking impulse activity by intravitreal injections 
of either TTX, a sodium-channel blocker, or 2-amino-4-phosphonobutyric acid (APB), a glutamate analog known to block ON-center channels (Horton, 1981; Slaughter and Miller, 1981; Schiller, 1982a, b; Knapp and Mistler, 1983). The latter manipulation was included because ON- and OFF-center cells are segregated to specific layers within the tree shrew LGN (Conway and Schiller, 1983; Holdefer and Norton, 1986). As the results will show, we found that neither of these manipulations prevented the development of LGN cell layers or altered retinal projection patterns, but that following complete blockade with TTX, interlaminar spaces were poorly differentiated and the entire LGN was slowed in its maturation. Some of these observations were reported previously (Casagrande et al., 1985).

\section{Materials and Methods}

Subjects. A total of 52 neonatal tree shrews (Tupaia belangeri) were used for this study. All of these animals were born in our colony and were hand-reared from the day of birth (P0). Survival periods ranged from a few days to a little over 2 weeks, depending on the experimental paradigm (see below). Previous work has shown that this period (which occurs before the day of lid-opening around P19) is critical for LGN laminar development. Specifically, all interlaminar spaces begin to develop between P0 and P8. By P14, most cytoarchitectonic and cytological aspects of LGN lamination appear mature (for reviews, see BrunsoBechtold and Casagrande, 1983; Casagrande and Brunso-Bechtold, 1985). On $\mathrm{P} 0$, weights and crown-rump lengths of all infants were recorded and, thereafter, relative changes in individual growth were monitored daily. When possible, littermates were used in matched control and experimental groups.

TTX injections. The sodium-channel blocker TTX was injected in total doses of $0.1-0.5 \mu \mathrm{g}$ in volumes of $0.6-3.0 \mu \mathrm{l}$. Injections were made into the vitreal chamber, either unilaterally or bilaterally, every other day from P0, for periods of a few days to over 2 weeks. Control pups were injected with the same volumes of vehicle (citrate buffer) or sterile distilled water. A total of 45 animals was used in this portion of the study: 17 received TTX injections bilaterally, 17 unilaterally, and 11 received control injections either bilaterally or unilaterally (see Table 1). In addition, the termination pattern of retinal projections in the LGN was examined in 6 of the TTX-treated pups and 2 controls by injecting one or both eyes with $3 \mu \mathrm{l}$ of $1 \%$ WGA conjugated to HRP (WGA-HRP) in 2\% dimethylsulfoxide (DMSO)-Tris buffer (0.05 M) 1 $\mathrm{d}$ before the animals were killed. Finally, in order to directly compare the effects of removing the retina with the effects of silencing action potential activity, 3 of the pups that received unilateral TTX eye injections also underwent enucleation of the other eye on P0.

For our injections, we surgically opened the fused lid of the eye(s) to be injected on P0 while the animal was cryogenically anesthetized, and placed a small hole at the limbus of the eye. The injection solution (dyed with methylene blue for easy visibility) was then introduced under microscopic guidance into the vitreal chamber of the eye, using a calibrated micropipette, tip diameter $30-60 \mu \mathrm{m}$, attached to a length of waterfilled polyethylene tubing. If significant back-lcakagc of solution was observed following an injection, the eye was reinjected with the estimated lost volume. Following the injection, the eye was covered with a protective coating of Neosporin ointment and the lid resutured. The pup was then placed on a heating pad and carefully monitored until it recovered. Subsequent injections were all carried out under the same anesthetic condition, and simply required the brief period necessary to remove the suture, visualize the location of the previously made small hole in the eye, and inject the eye.

For the enucleations, the fused lid of the eye of the anesthetized pup was first opened and a $1 \%$ solution of Alcaine or xylocaine was then dripped onto the eye and surrounding tissue. The eye itself was then gently freed of eye muscles and surrounding tissue, removed from the orbit, and replaced with a small piece of Gelfoam. The lid was then partially closed over the orbit with a single suturc.

$A P B$ injections. For these experiments, 5 pups were injected intravitreally daily with APB in $2-3 \mu$ l sterile saline, either unilaterally (cases $84-15,84-18$ ), or bilaterally (cases 84-16, 84-19, 84-62). Two pups $(84-14,84-17)$ served as controls and received unilateral injections of the same volume of vehicle (saline). Of the experimental animals, one
(84-62) received a dose of $330 \mu \mathrm{M} \mathrm{APB}, 2$ (84-15 and 84-16) received doses of $500 \mu \mathrm{M} \mathrm{APB}$, and $2(84-18,84-19)$ received doses of $2 \mathrm{~mm}$ APB. In adult tree shrews, the lowest doses in this range (i.e., 300-500 $\mu \mathrm{M}$ APB) were found to severely attenuate, but not completely block, ON-center cell activity recorded from the superior colliculus (T. T. Norton, personal communication). Dose schedules and amounts were based on effective protocols employed by others (Knapp and Mistler, 1983; A. G. Knapp and P. H. Schiller, personal communication; T. T. Norton, personal communication).

For our injections of APB, we employed surgical procedures similar to those described above for the TTX cases, except that chloral hydrate anesthesia ( $4 \mathrm{mg} / \mathrm{kg}$ body weight) was employed for pups older than 7 d. Pups survived until P5 (84-17, 84-18, 84-19), P6/7 (84-14, 84-15, $84-16$ ), and P14 (84-62). It is noteworthy that we found it difficult to keep pups alive using the above daily injection schedule. This problem was not due to the APB itself, since as many of our original controls died, as did experimental animals; rather, it likely reflected the added stress caused by daily, albeit brief, periods of anesthesia.

Histological procedures. At the end of the experiment, each pup was deeply anesthetized with chloral hydrate and perfused transcardially with a brief rinse of either warmed PBS or oxygenated lactated Ringer's followed by a longer perfusion of warmed fixative. Fixatives varied depending on requirements for tissue processing. In cases where sections were only stained for cell bodies, the pups were perfused with $10 \%$ buffered formalin. In cases where a combination of procedures was employed [e.g., sections reacted for cytochrome oxidase (CO) and/or HRP], the pups were perfused with $1-2 \%$ paraformaldehyde in combination with $1-2.5 \%$ glutaraldehyde in $0.1 \mathrm{M}$ phosphate buffer with or without $10 \%$ DMSO, followed by a brief rinse of $10 \%$ sucrose-fixative or $10 \%$ sucrose-buffer solution.

All brains were placed in $30 \%$ sucrose-fixative or sucrose-buffer and allowed to equilibrate before being frozen-sectioned horizontally at 30 $40 \mu \mathrm{m}$. Alternate sections through these brains were stained with cresyl violet, reacted for CO activity according to the protocol of Wong-Riley (1979), and if WGA-HRP had been injected into the eye, reacted with tetramethylbenzidine (Mesulam, 1976).

Examination of the retina and cell counts in the ganglion cell layer. In cases that involved eye injections, we systemically evaluated possible retinal damage. Following perfusion, each eye was marked for orientation, removed, and placed in Bouin's fixative. The anterior portion of the globe was subsequently removed and the retinal surface examined under the microscope for any gross changes in morphology. The eyecup containing the retina was then dehydrated, embedded in JB-4 plastic, and sectioned horizontally, alternately at 2 and $4 \mu \mathrm{m}$, from superior through inferior retina. Two adjacent sections (one 2 and one $4 \mu \mathrm{m}$ ) were saved every $60 \mu \mathrm{m}$ throughout the eye, except at the location of the optic disk, where sampling frequency was increased to 2 sections every $30 \mu \mathrm{m}$. All sections were then stained with $1 \%$ toluidine blue. All of these sections were then examined for gross morphological changes in retinal laminar structure or cytology.

Overall, the only evidence of any retinal abnormality occurred in 3 cases. In case 85-152, the left eye developed an infection that destroyed the retina. Not surprisingly, the results in this case (at the level of the LGN) were equivalent to those in the cases where one eye had been removed (see Results). In 2 cases (85-127, right retina, and 85-131, right rctina), pcculiar disruptions of the ganglion cell or other retinal cell layers were apparent in some sections. At these locations, segments of the inner nuclear and ganglion cell layers appeared transposed with respect to each other or appeared to be organized into rosettes. Cells within these retinal regions, however, exhibited normal morphology. These retinal laminar disruptions occurred in very limited regions of a few sections and did not appear to relate in any obvious way to LGN development.

In order to determine whether the injection procedures affected the relative frequency of cells located in the ganglion cell layer in the TTX experimental group, counts were made at $200 \times$ magnification in all sections that contained the optic disk. In these sections, all cells in the ganglion cell layer within every other $0.38 \mathrm{~mm}$ stretch of temporal retina were counted. With this sampling procedure, an average of 5 zones was counted on 10-15 sections. We felt that the above sampling procedure was appropriate because it allowed us to (1) be certain that we were sampling from the same regions in all eyes, a necessity in retinae where the ganglion cell distribution is nonuniform and/or changing with development; (2) sample a broad region of the binocular segment of the retina, all of which lies temporal to the optic disk in tree shrews; (3) 
Table 1. Control and TTX cases: procedures and survival days

\begin{tabular}{llllll} 
Case & Procedure & $\begin{array}{l}\text { Sur- } \\
\text { vival }\end{array}$ & Case & Procedure & $\begin{array}{l}\text { Sur- } \\
\text { vival }\end{array}$ \\
\hline $84-29$ & TTX (B) & P13 & $85-84$ & Cont (B) & P14 \\
$84-41$ & TTX (R) & P12 & $85-88$ & TTX (B) & P05 \\
$84-45$ & Cont (R) & P14 & $85-89$ & TTX (R) & P06 \\
$84-46$ & Cont (L) & P14 & $85-90$ & Cont (B) & P06 \\
$84-47$ & TTX (L) & P14 & $85-105$ & TTX (B) & P18 \\
$84-48$ & TTX (B) & P14 & $85-106$ & TTX (R) & P14 \\
$84-49$ & TTX (R) & P14 & $85-107$ & Cont (R) & P02 \\
$84-58$ & TTX (R) & P04 & $85-114$ & TTX (B) & P08 \\
$84-59$ & TTX (B) & P08 & $85-115$ & TTX (L) & P13 \\
$84-61$ & Cont (R) & P07 & $85-116$ & Cont (R) & P13 \\
$85-60$ & TTX (B) & P05 & $85-117$ & TTX (R) & P06 \\
$85-61$ & TTX (L) & P07 & $85-126$ & TTX/WGA-HRP (B) & P15 \\
$85-62$ & Cont (R) & P24 & $85-127$ & TTX (B)/WGA-HRP (R) & P15 \\
$85-70$ & TTX (B) & P14 & $85-130$ & EN (R)/TTX (L)/WGA-HRP (L) & P16 \\
$85-71$ & TTX (L) & P15 & $85-131$ & EN (L)/TTX (R)/WGA-HRP (R) & P16 \\
$85-72$ & Cont (L) & P15 & $85-132$ & EN (R)/TTX (L)/WGA-HRP (L) & P16 \\
$85-73$ & TTX (B) & P15 & $85-152$ & TTX (B)/WGA-HRP (B) & P15 \\
$85-70$ & TTX (B) & P17 & $85-153$ & TTX (B) & P11 \\
$85-80$ & TTX (L) & P16 & $85-191$ & TTX (B)/WGA-HRP (B) & P14 \\
$85-81$ & Cont (L) & P17 & $85-194$ & TTX (R)/WGA-HRP (R) & P15 \\
$85-82$ & TTX (B) & P03 & $85-11$ & Normal uninjected & P14 \\
$85-83$ & TTX (R) & P09 & $85-16$ & Normal uninjected & P14 \\
\hline
\end{tabular}

TTX, Eye injection with tetrodotoxin; Cont, eye injection with vchiclc; WGA-HRP, cyc injection with wheat germ agglutinin conjugated to horseradish peroxidase; EN, eye enucleation at P0; B, bilateral eye injection; L, left eye injection; $\mathrm{R}$, right eye injection; $\mathrm{P} 0$, day of birth; $\mathrm{P}$, postnatal day.

sample in retinal regions that normally project to the most well-defined laminated portions of the LGN.

Relative frequency and distribution of cells in the ganglion cell layer were compared for matched samples from the left and right eyes of individual cases, and for samples collapsed across each retina. In addition, collapsed samples for experimental and control eyes were compared.

The results of these comparisons demonstrated that TTX did not significantly affect cell number in the ganglion cell layer. Comparisons between cases, as well as between all control and TTX-injected eyes demonstrated that the cell frequency within sampling areas was unaffected by TTX treatments. TTX-injected eyes had a mean cell frequency of $63.7 \pm 0.8(\mathrm{SEM})$, whereas injected control eyes and uninjected control eyes had a mean cell frequency of $64.1 \pm 0.8$. The differences between the distributions for TTX and control eyes were not statistically significant using the chi-square distribution: $13.3(p<0.20)$. We conclude from these analyses that repeated injections of TTX for 1 or 2 weeks do not significantly affect the frequency or relative distribution of cells in the ganglion cell layer. It is, therefore, unlikely that the TTX injections differentially affected the ganglion cells that make up the majority of neurons in the ganglion cell layer, the remainder being displaced amacrine cells (Perry, 1982). Thus, the changes in LGN laminar organization reported below are related to modifying action potential activity and not to a reduction in ganglion cells. However, we cannot rule out the possibility that the TTX treatments affected ganglion cell size, a result that has been reported in cats but not in frogs following eye injections (Reh and Constantine-Paton, 1985; Dubin et al., 1986). Regardless, demonstration of relatively smaller retinal ganglion cells following TTX treatment in the tree shrew would simply reinforce our conclusion that the main effect of blocking activity with TTX is to slow maturation (see Results).

Soma size analysis of $L G N$ layers. In order to evaluate the relative effects of TTX treatments on the development of LGN cells, we sampled cells from each layer of 9 P12-16 tree shrews listed in Table 1. In normal tree shrews, soma size measures do not show statistically significant differences in cell size between these ages. A minimum of 50 cells/layer was sampled from both ipsilateral and contralateral LGNs of unilateral control injected and TTX-injected animals. All animals used for soma size analyses were perfused with mixed aldehydes, as noted above. For bilateral TTX-injected animals, we sampled cells from each set of matched layers $1-2$ and 4-5, in addition to layers 3 and 6 . These samples were compared to matched control layers $1-2$ and 4-5. A total of 4487 cells from normal uninjected, control injected, and TTX-injected animals were drawn, using a $100 \times$ oil-immersion objective with the aid of a drawing tube, and were differentiated from glia by standard criteria. All sampling areas were taken from the binocular segment of the LGN in sections that contain the optic disk representation. Cell area measures and comparisons were made using the Bioquant System IV Image Analysis System (E. Leitz).

Visual evoked potential recording. To insure that the doses and schedule of TTX injections effectively blocked neural activity, we examined visual evoked potential (VEP) responses recorded from the striate cortex and/or superior colliculus of adult and infant tree shrews of various ages, from P0 to P30. The most satisfactory results were obtained by recording from lightly anesthetized animals (Ketamine and Nembutal in adults, and chloral hydrate in infants). Animals were stabilized, either in a stereotaxic apparatus by a standard head-holder (adults), or by gluing the skull to a specially designed carrier (infants). In each case, pupils were dilated with $1 \%$ atropine and the corneas protected with a thin film of silicone. VEPs were recorded using unipolar electrodes (David Kopf; tip diameter, $0.5 \mathrm{~mm}$ ) with an indifferent reference. VEPs were evoked by stroboscopic flash at $1 \mathrm{~Hz}$ for adults and $0.5 \mathrm{~Hz}$ for younger tree shrews. For each series, 5 min of VEP trials were averaged with an Enhancetron signal-averager and recorded on a chart recorder. Sweep time was $250-500 \mathrm{msec}$ synchronous with the light flash onset. Control trials of equal duration were recorded after VEP trials by masking both test and control eyes. In each case, VEP components produced by each eye, in each hemisphere, were evaluated prior to TTX injections. Thirty to $60 \mathrm{~min}$ following TTX eye injections at doses ranging from 0.1 to $0.4 \mu \mathrm{g}$, VEPs were again evaluated. In a number of cases, we then allowed the animal to recover and repeated the VEP recordings 2,3 , or $4 \mathrm{~d}$ later.

Since many of the younger infants did not survive the recording sessions, we were not able to evaluate the effectiveness of our TTX 


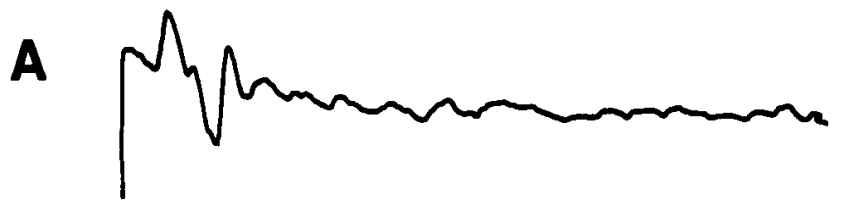

B

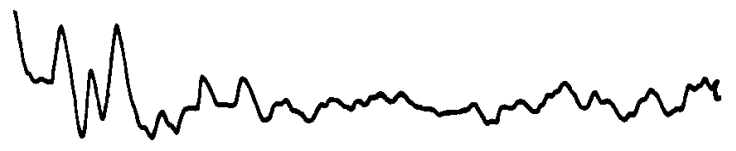

C

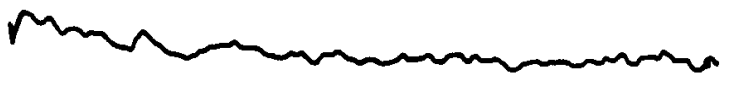

D

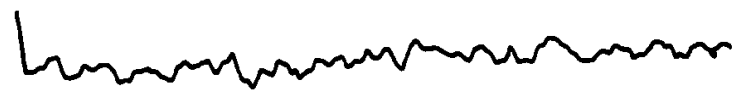

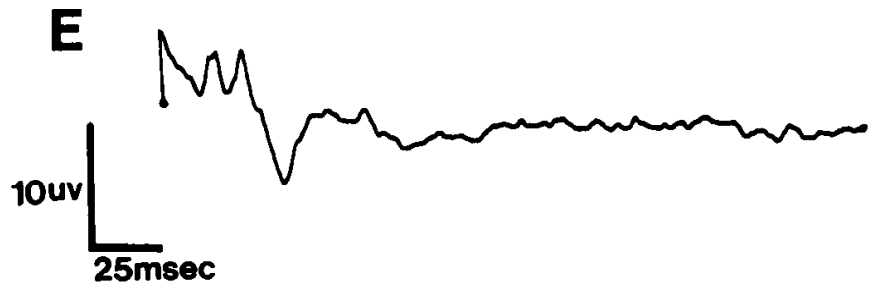

Figure 1. Visual evoked potential (VEP) recordings from an adult tree shrew striate cortex, showing the effectiveness of TTX action potential blockade. $A$, The initially normal VEP from the right cortex averaged over 300 trials. This animal later received a single intraocular injection of $0.4 \mu \mathrm{g}$ of TTX into the left eye. $B$, Normal VEP recorded from the left cortex while stimulating the right uninjected eye $2 \mathrm{~d}$ following TTX injection. Note the normal biphasic shape of the VEP. $C$, No VEP could be recorded contralateral to the TTX-injected eye $2 \mathrm{~d}$ after TTX injection, demonstrating that the TTX injection had action potential blocking effects for at least 2 d. $D$, Control record with no lights. $E$, VEP recorded at the same cortical location seen in $C$ and $D$, elicited through the ipsilateral (uninjected) eye.

injections for each experimental animal throughout its series of injections. Further, pupillary responses (see Dubin et al., 1986) were not found to be a satisfactory indicator of neural blockade, since it was impossible to reliably detect changes in pupil size in normal tree shrews even at P14, which is still 3-5 d before natural lid-opening. Therefore, all of our evaluations of the effectiveness of the TTX blockade were limited to recordings made on a separate series of infants and adults. The earliest age at which we were able to successfully complete the series of VEP recordings over the $2 \mathrm{~d}$ necessary to demonstrate the effects of TTX was $P 6 / 7$. Evoked potentials at this age (not illustrated) are small, immature, and exhibit a single low-latency peak; VEPs in older pups exhibit a more mature biphasic pattern.

Our most reliable recordings were obtained from older infants and from adult animals. In these cases, doses of $0.1-0.15 \mu \mathrm{g}$ of TTX (the lowest doses used in our experimental series) were sufficient to completely and reliably block VEPs recorded from the treated eye for more than $2 \mathrm{~d}$. Our results also showed that the TTX blockade became ineffective 3-4 d postinjection, in which case normal VEPs could again
A
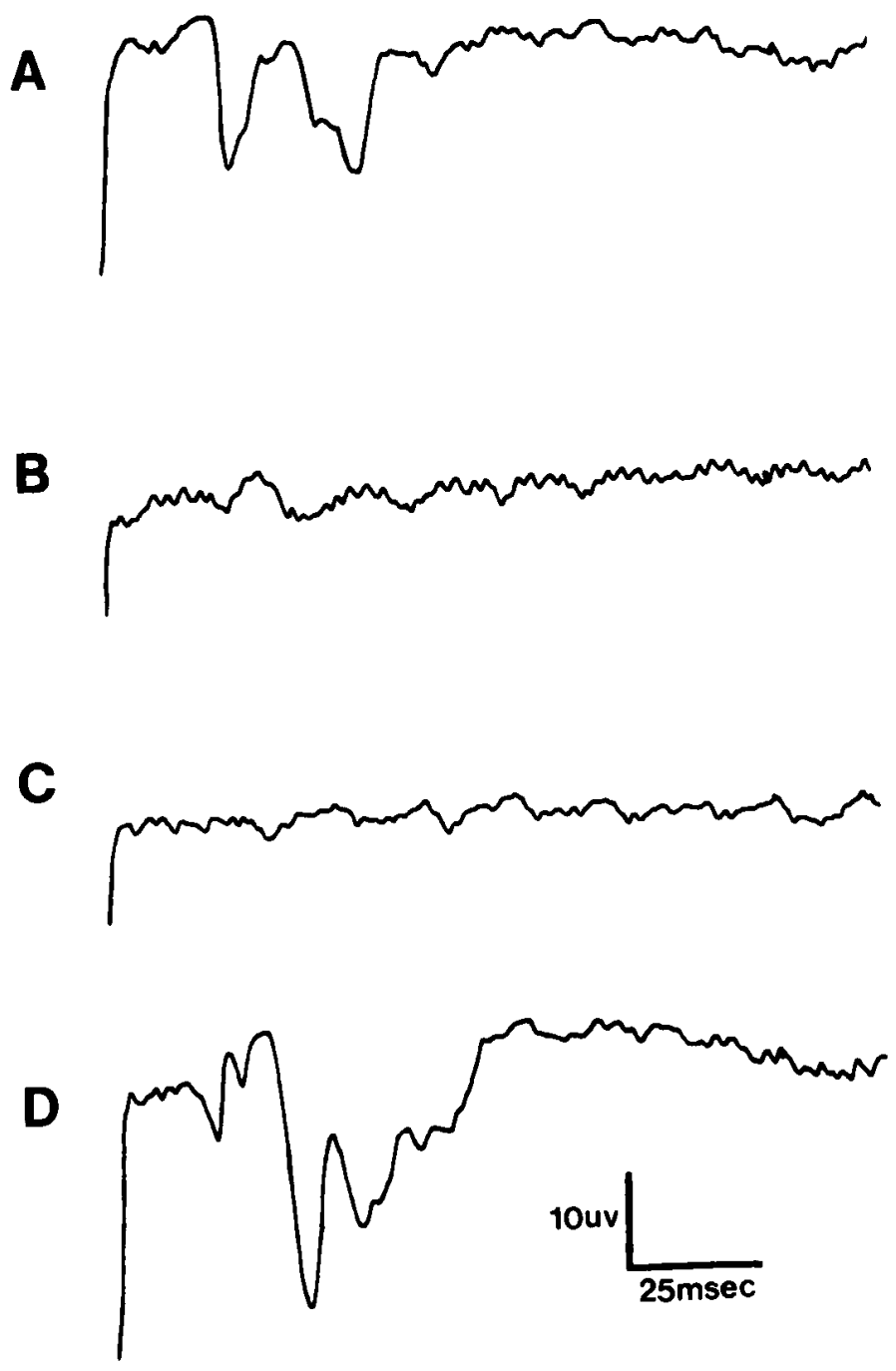

Figure 2. Visual evoked potential (VEP) recordings from a P30 tree shrew. $A$, Normal VEP recorded in right striate cortex following stimulation of the left eye, averaged over 166 trials. This animal subsequently received a single intraocular injection of $0.15 \mu \mathrm{g}$ of TTX into the left eye. $B$, VEP recorded contralateral (right striate cortex) to the TTXinjected eye $30 \mathrm{~min}$ following the injection, indicating that the lowest TTX dosages used for our experiments effectively blocked action potential activity. $C$, Control record with no light. $D$, VEP recorded at the same cortical location as in $B$, elicited through the ipsilateral (uninjected) eye.

be obtained. Figures 1 (adult) and 2 (infant) show examples of VEP records from striate cortex prior to and following TTX injections. As may be noted in both cases, records from the cortex contralateral to the TTX-injected eye show no evidence of VEP responses, yet normal ipsilateral components are obvious from the uninjected eye.

\section{Results}

In our analyses of the effects of altered neural activity, comparisons were made with controls, using the representation of the optic disk (see Fig. $5 J$ ) as an anchor point to match sections between cases. For the purpose of illustration, we selected all LGN sections at or near the optic disk representation and oriented all photomicrographs of LGN sections the same way, with anterior toward the bottom and medial toward the right of the illustration. 

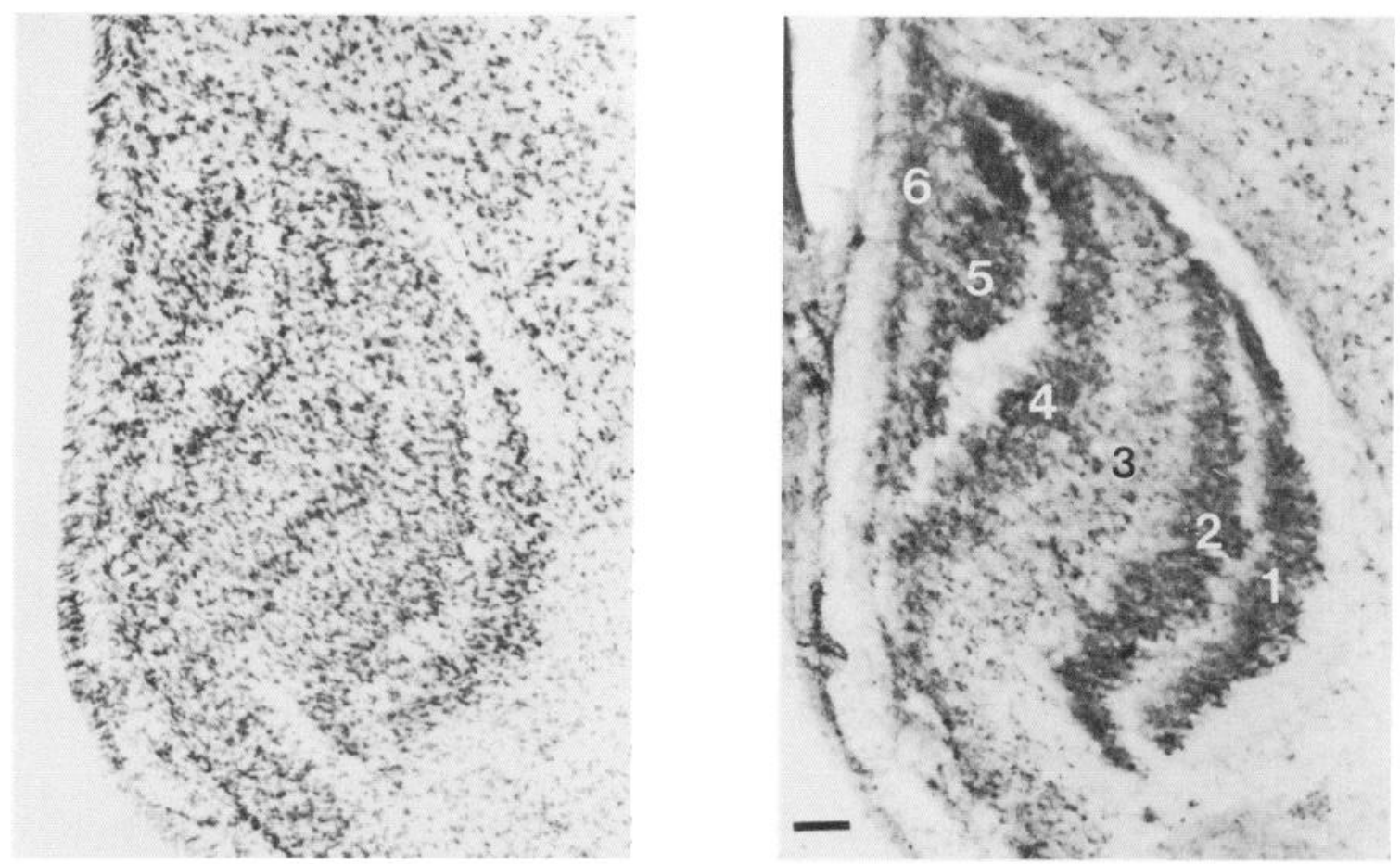

Figure 3. Nissl- (left) and CO- (right) stained horizontal sections through the LGN of a P14 tree shrew (case 84-45), which served as a control. Posterior towards the top and lateral towards the left of each photomicrograph. The sections shown are ipsilateral to the citrate buffer-injected left eye. In tree shrews, 4 layers receive contralateral retinal input $(2,3,4$, and 6$)$ and 2 layers receive ipsilateral retinal input (1 and 5$)$. The overall appearance of control and normal P14 LGNs was the same. See text for details. Scale bar, $0.1 \mathrm{~mm}$.

\section{Effects of TTX on cellular lamination and metabolic activity}

Normal and control cases. At P14, the tree shrew LGN is welllaminated, although the interlaminar spaces between cell layers are somewhat narrower and more populated with cells, and the volume of the whole nucleus is smaller than that of the adult. Figure 3 shows an example of the appearance of sections through the nucleus in a typical injected control case stained for Nissl substance (Fig. 3, left) and reacted for CO activity (Fig. 3, right).

The layer 1-2 and 4-5 interlaminar spaces are normally the most obvious in Nissl-stained material at P14. Furthermore, the relative differences in cell size and staining (cytological characteristics of lamination) that are evident in adult tree shrews are seen by P14, e.g., the cells in layer 3 are smaller (see cell size analysis below) and paler than those in adjacent layers. Interlaminar spaces defined metabolically in CO-stained sections are often more obvious than those in adjacent Nissl-stained sections. Finally, as reported by Norton and Wong-Riley (1984), our results show that metabolic activity is not equivalent across layers: layer 3, and, to a lesser extent, layer 6 tend to show less $\mathrm{CO}$ activity than do the remaining 4 layers.

The appearance of LGN layers in normal and control injected cases was not qualitatively different in Nissl-stained or COreacted sections. However, quantitative soma size analysis did show a small effect of control injections on cell size.

Analysis of soma size distributions in all layers of the LGN is shown in Figure 4 and summarized in Table 2. For this analysis, we compared LGN layers innervated by normal, uninjected eyes and layers innervated by control injected eyes in 2 control animals as a basis for our analysis of the main effects of TTX treatments on the LGN. Comparisons (not shown) between soma sizes in the LGNs of normal tree shrews and those in LGN cells innervated by uninjected eyes in control tree shrews showed no statistical difference. As may be noted in Figure 4 and Table 2, unilateral control eye injections did result in some significant changes in LGN soma size: maximal for layers 2 and 5 , and minimal, but still significant, for layers 1 and 6 . Although the mean differences between control innervated layers were statistically significant for layers $1,2,5$, and 6 , the variance in the overall soma size distributions for those comparisons accounted for only $2.3-10.6 \%$ of the distribution variances. Nonetheless, for our analysis of the effects of TTX treatments on LGN soma size, reported in the following 2 sections, we used the laminar soma size distributions from layers innervated by control injected eyes to provide a conservative estimate of the

\begin{tabular}{|c|c|c|c|c|c|}
\hline \multirow[b]{2}{*}{$\underline{\text { Layer }}$} & \multicolumn{2}{|c|}{ Mean soma area $\left(\mu \mathrm{m}^{2} \pm \mathrm{SD}\right)$} & \multirow[b]{2}{*}{$t$ Ratio } & \multirow[b]{2}{*}{$d f$} & \multirow[b]{2}{*}{$\begin{array}{l}\text { Omega } \\
\text { square }\end{array}$} \\
\hline & $\begin{array}{l}\text { Control } \\
\text { uninnervated }\end{array}$ & $\begin{array}{l}\text { Control } \\
\text { innervated }\end{array}$ & & & \\
\hline 1 & $168.7 \pm 50.5$ & $153.3 \pm 41.1$ & $2.5^{*}$ & 217 & 0.023 \\
\hline 2 & $164.4 \pm 40.6$ & $137.9 \pm 36.4$ & $5.3^{* *}$ & 236 & 0.106 \\
\hline 3 & $131.0 \pm 35.3$ & $131.8 \pm 37.3$ & $0.2^{a}$ & 216 & 0.004 \\
\hline 4 & $149.9 \pm 43.1$ & $151.2 \pm 45.7$ & $0.2^{a}$ & 221 & 0.004 \\
\hline 5 & $182.3 \pm 50.5$ & $153.2 \pm 49.9$ & $4.7^{* *}$ & 257 & 0.075 \\
\hline 6 & $147.0 \pm 45.9$ & $162.3 \pm 45.8$ & $2.5^{*}$ & 216 & 0.024 \\
\hline
\end{tabular}

\footnotetext{
${ }^{a}$ Nonsignificant.

$* p<0.02 ; 2$-tailed $t$ test.

$* * p<0.001 ; 2$-tailed $t$ test.
} 

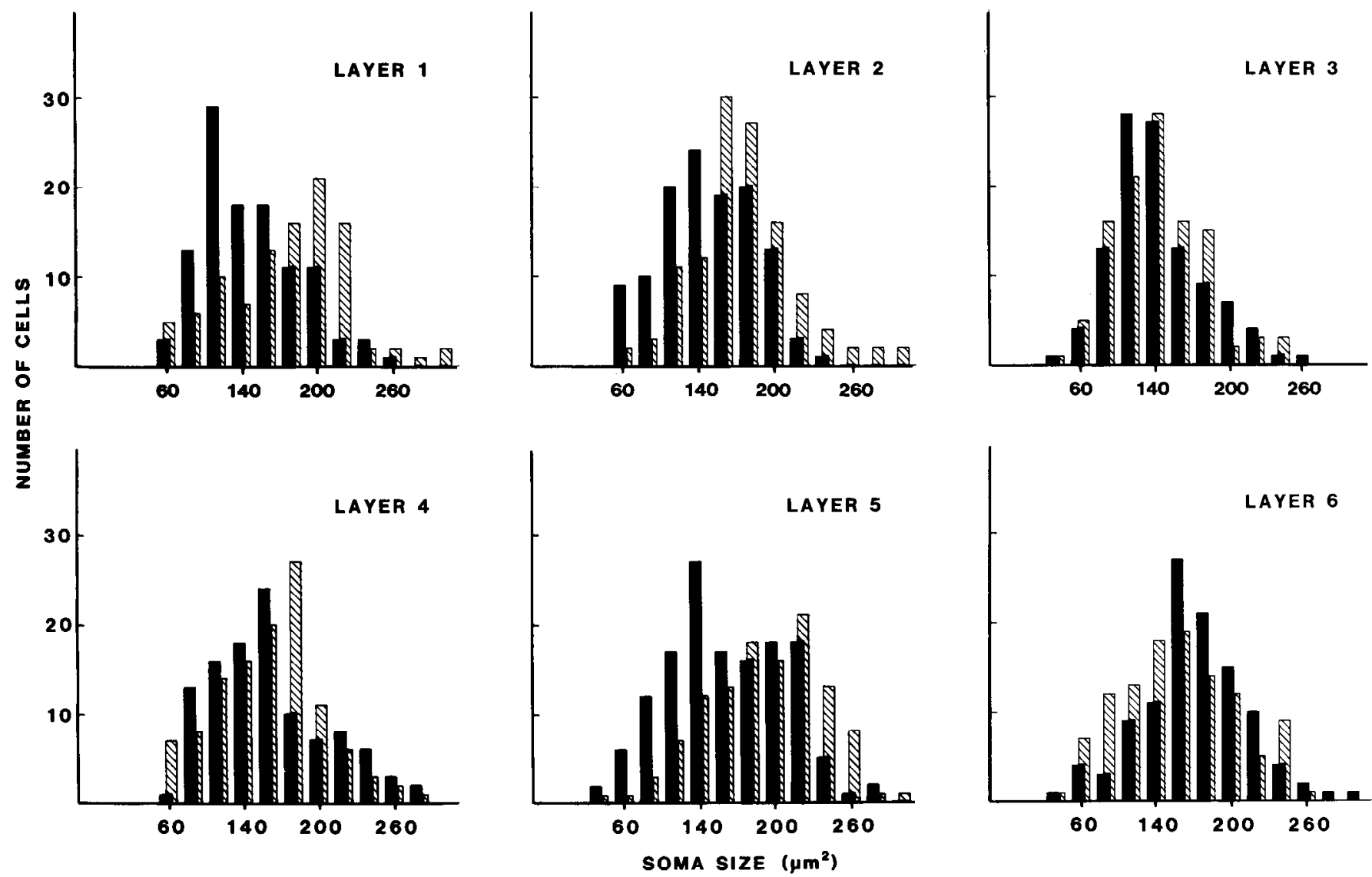

Figure 4. Soma size distributions for LGN layers innervated by control injected eyes (filled) compared to LGN layers innervated by uninjected eyes (cross-hatched). As noted in the text and in Table 2, layers innervated by vehicle-injected eyes showed a small but significant change in soma area for LGN layers $1,2,5$, and 6 (mean soma sizes and statistical comparisons between control uninnervated and control innervated are listed in Table 2). The significant mean differences between control innervated layers and uninnervated LGN layers account for a small proportion of the overall soma size variance. However, since some significant differences were found between layers, all comparisons for TTX effects presented used the control innervated layer soma size distributions presented here for comparison with TTX-innervated layers.

main effects of TTX treatments on LGN laminar formation and cell size.

Bilateral TTX injections. In contrast to the normal appearance of the LGN in the control animals, the LGNs of tree shrews that had both eyes injected with TTX for 14 or more days are small and poorly laminated. Figure 5 shows examples of this result in 5 animals. For each experimental case, adjacent $\mathrm{CO}$ and Nissl-stained LGN sections are shown.

Several structural effects are found in animals that received bilateral TTX injections. Most important, in Nissl-stained sections, some interlaminar spaces are evident in all cases (i.e., cytoarchitectonically defined lamination does develop), and cytological characteristics of specific layers do develop in most cases (e.g., layer 4 cells develop dark-staining cellular leaflets and layer 3 cells appear lighter-staining). The nucleus in every case, however, appears immature, smaller, and layers are less well-differentiated. Qualitatively, interlaminar spaces are narrow in comparison to those of normal animals of comparable age. In fact, animals bilaterally injected with TTX for $l$ week or less show no evidence of interlaminar space development (i.e., no evidence of cytoarchitectonic lamination), in contrast to 14-d-old control and TTX animals, in which some interlaminar spaces are always present. Quantitatively, as shown in Fig- ure 6 and Table 3A, all LGN layers from the 3 bilateral 2-weekold TTX-treated animals used for soma size comparisons show significantly smaller mean soma sizes that do controls $(p<0.001$ on a 2 -tailed $t$ test for all laminar comparisons). Further, these mean differences account for $26.7-53.5 \%$ of the variance between experimental and control conditions (see omega square values, Table $3 \mathrm{~A}$ ), suggesting a reliable main effect of TTX treatments.

It is noteworthy that bilateral TTX eye injections for 2 weeks show variable and, in certain cases, asymmetrical effects on the layers. The pattern of asymmetry shown in Figures $5, E, F$ (where layers innervated by one TTX-treated eye appear narrower and more lightly stained in $\mathrm{CO}$ and Nissl sections, respectively) most likely reflects an asymmetry in the effectiveness of the treatment. However, the more consistent differences between the relative differentiation of layers 1 and 2 and the remainder of the nucleus in TTX-treated cases suggest that TTX blockade does have more of an effect on some LGN layers than on others. This difference can best be appreciated by comparing the poorly developed layer 1-2 interlaminar space (see right arrows, Fig. 5) with the relatively well-developed layer 4-5 interlaminar space (left arrows, Fig. 5).

Nonetheless, it should be noted that some aspects of LGN 
laminar development remain relatively immune to bilateral TTX treatment. As shown in Figure 5, $B, D, F, H, J$, the relative differences in CO-staining intensity between LGN layers appear to develop normally (e.g., layer 3 is consistently lighter-staining than neighboring layers). Further, lamination is generally more apparent in CO-stained sections than in Nissl-stained sections (compare Fig. 5, $I$ and $J$ ), as is the case for the normal LGN (see Fig. 3), but the difference between lamination revealed in CO-stained and Nissl-stained material is exaggerated.

Unilateral TTX injections. Figures 7-9 show examples of 3 cases in which tree shrews received unilateral TTX injections for 2 weeks. Although the effects of unilateral TTX eye injections tend to be more variable than those of bilateral injections, all cases of unilateral eye injection show an asymmetry in LGN laminar development. In all unilateral TTX cases, layers innervated by the TTX-treated eye are consistently (1) more lightly stained in both Nissl and CO material, (2) narrower, and (3) made up of smaller cells than are their normally innervated counterparts.

The most extreme example of this effect is shown in Figure 7 , in which the left eye was injected with TTX (the layers innervated by this eye are indicated by numerals). In Nissl-stained sections, ipsilaterally innervated layers 1 and 5 are very narrow (Fig. $7 A$ ), while contralaterally (Fig. $7 B$ ), the effect is less dramatic, but still distinct. In CO-reacted sections from this case (Fig. 7, $C, D$ ), the TTX-affected layers are much lighter. The only exception is that the outer leaflet of layer 4 , contralateral to the TTX-injected eye (arrow in Fig. 7D), appears to maintain normal staining. At present, we have no explanation for the differential effect of TTX on the 2 leaflets of layer 4 . This result hints at the existence of, as yet, unidentified functional differences between the 2 leaflets of LGN layer 4 .

As with bilateral TTX eye injections, the relative development of layers 1 and 2, and the interlaminar space between them, appears more affected by TTX treatment than does that of the remainder of the nucleus in both Nissl and $\mathrm{CO}$ material (see Fig. 9B). Further, unilateral TTX eye injections often result in a more normal appearance of lamination in CO-stained sections than in the Nissl-stained sections. An example of this can be seen in case 85-115 (Fig. 9). In this case the CO staining of the layers looks relatively normal, except for a tendency for layers 1 and 5 ipsilateral to the eye injected (Fig. $9 D$ ) to stain more lightly. In contrast, cytoarchitectonic lamination in the Nissl-stained sections from this case is indistinct, especially contralateral to the TTX eye injection (Fig. 9B).

In some respects, the effect of unilateral TTX cyc injections resembles the effect seen following monocular enucleation in an adult tree shrew, that is, both monocular and binocular segments of the nucleus appear to be affected by the treatment (Casagrande et al., 1978). This can be seen clearly in Figure 7 (see also Fig. 8 ), where the "deprived" (or TTX-affected) contralaterally innervated layers show less $\mathrm{CO}$ activity throughout the nucleus (Fig. $7 D$ ) than their normally innervated counterparts (Fig. 7C).

Analysis of the soma size distributions from 4 unilaterally injected TTX animals is shown in Figure 10 and summarized in Table 3B and C. As may be noted in the soma size histograms in Figure 10, all LGN layers innervated by TTX-injected eyes show statistically significant changes compared to LGN layers innervated by vehicle-injected control eyes (all laminar comparisons are significant beyond the $p<0.001$ level on a 2-tailed $t$ test). Further, these mean differences account for $23.2-39.0 \%$ of the overall variance between TTX-and control innervated
Table 3. TTX soma size analysis: layers innervated by control injected eyes compared to layers innervated by TTX-injected eyes

\begin{tabular}{|c|c|c|c|c|c|}
\hline \multirow[b]{2}{*}{ Layer } & \multicolumn{2}{|c|}{ Mean soma area $\left(\mu \mathrm{m}^{2} \pm \mathrm{SD}\right)$} & \multirow[b]{2}{*}{$\begin{array}{l}t \\
\text { Ratio* }\end{array}$} & \multirow[b]{2}{*}{$d f$} & \multirow[b]{2}{*}{$\begin{array}{l}\text { Omega } \\
\text { square }\end{array}$} \\
\hline & $\begin{array}{l}\text { TTX- } \\
\text { innervated }\end{array}$ & $\begin{array}{l}\text { Control- } \\
\text { innervated }\end{array}$ & & & \\
\hline \multicolumn{6}{|c|}{ A. Bilateral TTX } \\
\hline $1-2$ & $79.6 \pm 28.9$ & $145.5 \pm 39.5$ & 20.4 & 459 & 0.474 \\
\hline $4-5$ & $102.0 \pm 34.0$ & $152.4 \pm 47.8$ & 13.3 & 481 & 0.267 \\
\hline 3 & $74.0 \pm 23.7$ & $131.8 \pm 37.3$ & 14.6 & 240 & 0.467 \\
\hline 6 & $100.7 \pm 40.8$ & $162.3 \pm 45.8$ & 15.8 & 214 & 0.535 \\
\hline \multicolumn{6}{|c|}{ B. Unilateral TTX } \\
\hline 1 & $99.1 \pm 28.7$ & $153.3 \pm 41.1$ & 11.1 & 217 & 0.358 \\
\hline 2 & $94.9 \pm 31.1$ & $137.9 \pm 36.4$ & 9.7 & 232 & 0.285 \\
\hline 3 & $88.5 \pm 24.0$ & $131.8 \pm 37.3$ & 10.2 & 215 & 0.322 \\
\hline 4 & $105.9 \pm 34.7$ & $151.2 \pm 45.7$ & 8.0 & 206 & 0.232 \\
\hline 5 & $105.6 \pm 24.1$ & $153.2 \pm 49.4$ & 9.0 & 242 & 0.247 \\
\hline 6 & $97.1 \pm 33.9$ & $162.3 \pm 45.8$ & 11.8 & 214 & 0.390 \\
\hline \multicolumn{6}{|c|}{ C. Unilateral TTX-matched sets of layers } \\
\hline $1-2$ & $96.9 \pm 30.0$ & $145.5 \pm 39.5$ & 14.6 & 451 & 0.319 \\
\hline $4-5$ & $105.8 \pm 29.7$ & $152.4 \pm 47.8$ & 12.1 & 450 & 0.243 \\
\hline
\end{tabular}

$* p<0.001 ; 2$-tailed $t$ test for all laminar comparisons.

LGN layers (see estimated omega square values in Table 3B and C). As noted above (see also Discussion), TTX blockade also appears to affect layers 1 and 2 more severely than it does layers 4 and 5 . This is quantitatively demonstrated in the comparisons of matched sets of LGN layers seen in Figure 11 and summarized in Table 3C. Specifically, the soma size distributions of layers 1 and 2 show a more dramatic change in soma area compared to matched sets from control injected cases. In fact, $31.9 \%$ of the overall variance in the soma size distributions for layers 1 and 2 can be directly related to the TTX treatments, whereas, $24.3 \%$ of the mean difference between layers 4 and 5 from TTX cases, compared to control layers 4 and 5 , can be accounted for by TTX treatments.

\section{Effects of TTX on retinal projections}

Figure 12 shows sections from the left (Fig. 12A) and right (Fig. $12 B$ ) LGN in a normal P14 tree shrew that received a WGAHRP injection in both eyes at P13 and was allowed to survive to P14. As in adult tree shrews (Casagrande and Brunso-Bechtold, 1983, 1985), the segregation of retinal input from the 2 eyes is unequal across the nucleus and does not entirely reflect the spacing between cell layers. This is especially obvious when one compares the very small space between the distributed ipsilateral and contralateral retinal afferents in layers 1 and 2 with the wide space between the ipsilateral and contralateral retinal afferents in layers 4 and 5: both spaces are equally well-developed in a Nissl stain (see Fig. 3). Further, contralaterally innervated layers 3 and 6 receive a lighter retinal input than do their contralaterally innervated neighboring layers 2 and 4 , as they do in adult tree shrews (McKanna and Casagrande, 1985).

Figure $12 C, D$, shows the left and right LGNs of case 8591, which received a 2 week regimen of binocular TTX injections, as well as binocular injections of WGA-HRP $1 \mathrm{~d}$ prior to being killed on P14. The labeled projection appears welllaminated. In none of the binocular TTX cases, where projections were traced from one or both eycs, is there cvidence to 

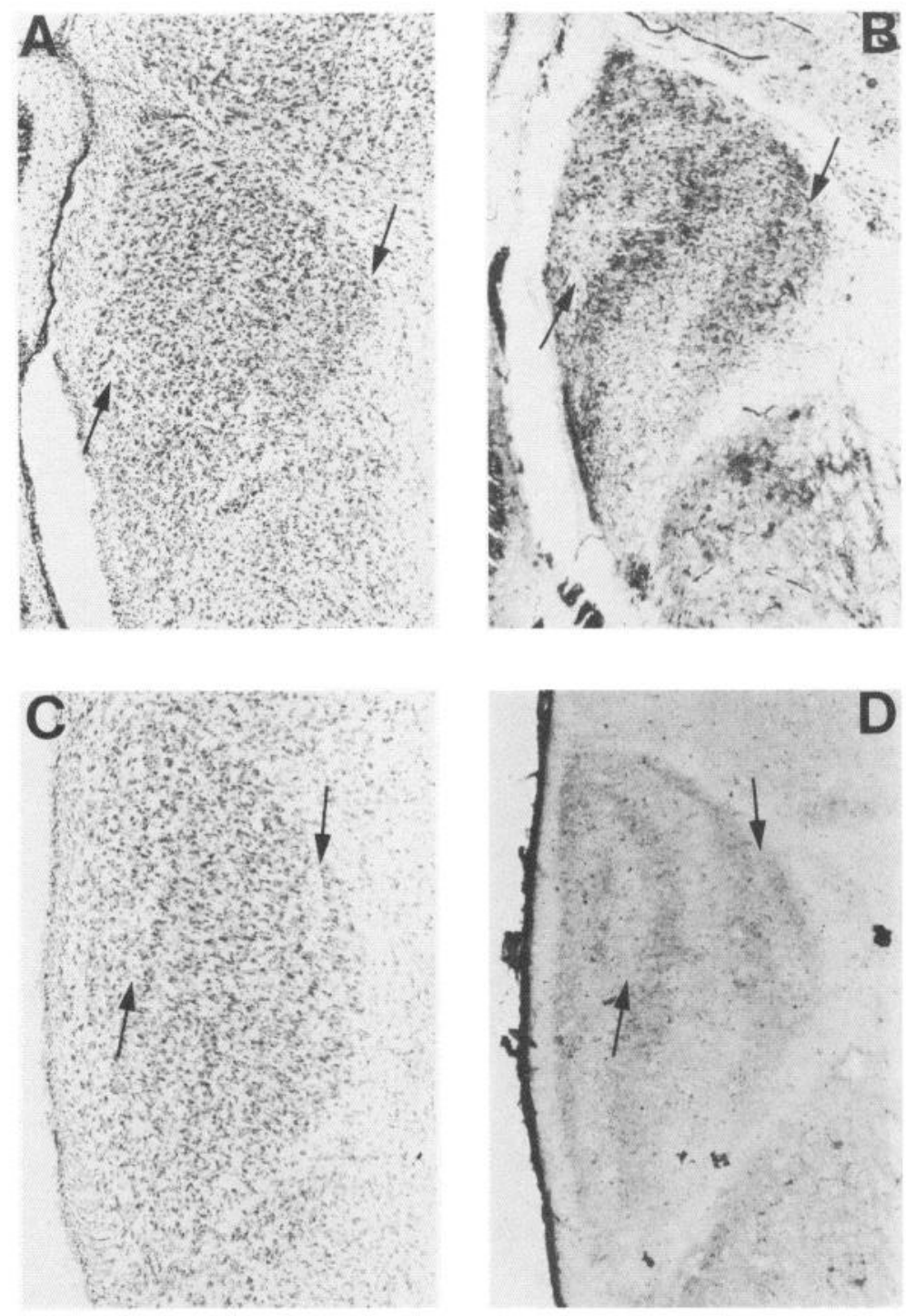

Figure 5. Nissl- $(A, C, E, G, I)$ and CO- $(B, D, F, H, J)$ stained horizontal sections through the LGNs of 5 tree shrews that received bilateral TTX eye injections for 2 weeks or more. Posterior is towards the top and lateral towards the left of each photomicrograph. The LGN in each of these cases resembles that of an immature animal. Compare the general degree of laminar development shown here with that shown in Figure 3. $A, B$, Adjacent sections from case 85-70 (P14). $C, D$, Sections from case 85-126 (P15). E, F, Adjacent sections from case 84-48 (P14). $G, H$, Adjacent sections from case 85-79 (P17). $I, J$, Sections from case 85-73 (P15). Small arrows indicate the location of the layer 1 and 2 (right) and layer 4 and 5 (left) interlaminar spaces. Large arrowhead in $J$ indicates the position of the optic disk representation. Numerals refer to the LGN layers. See text for details. Scale bar, $0.1 \mathrm{~mm}$.
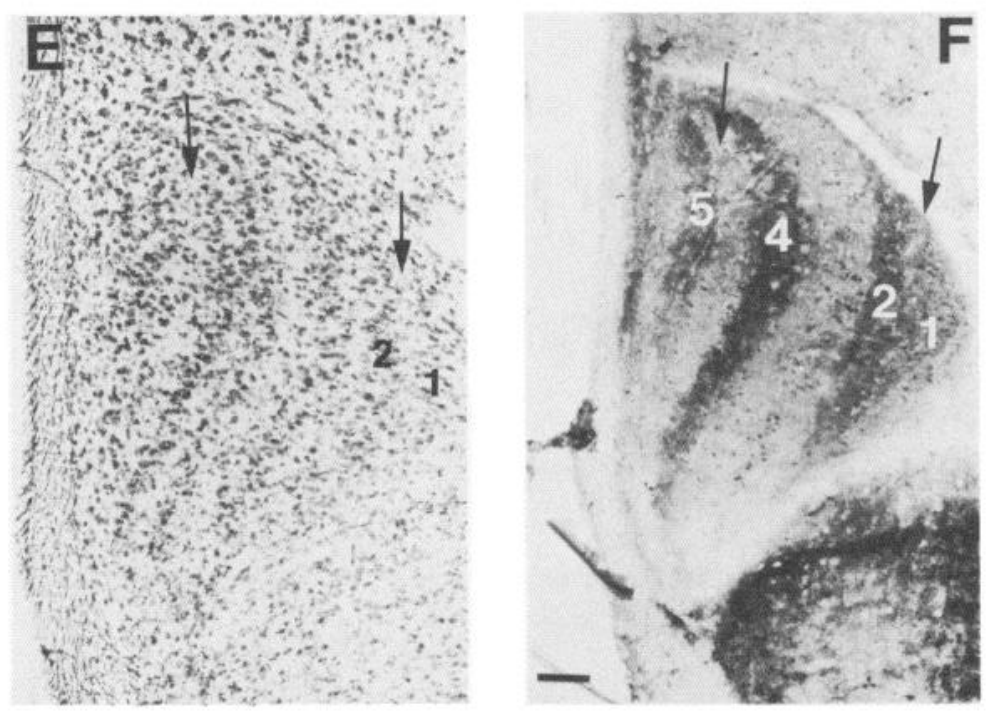

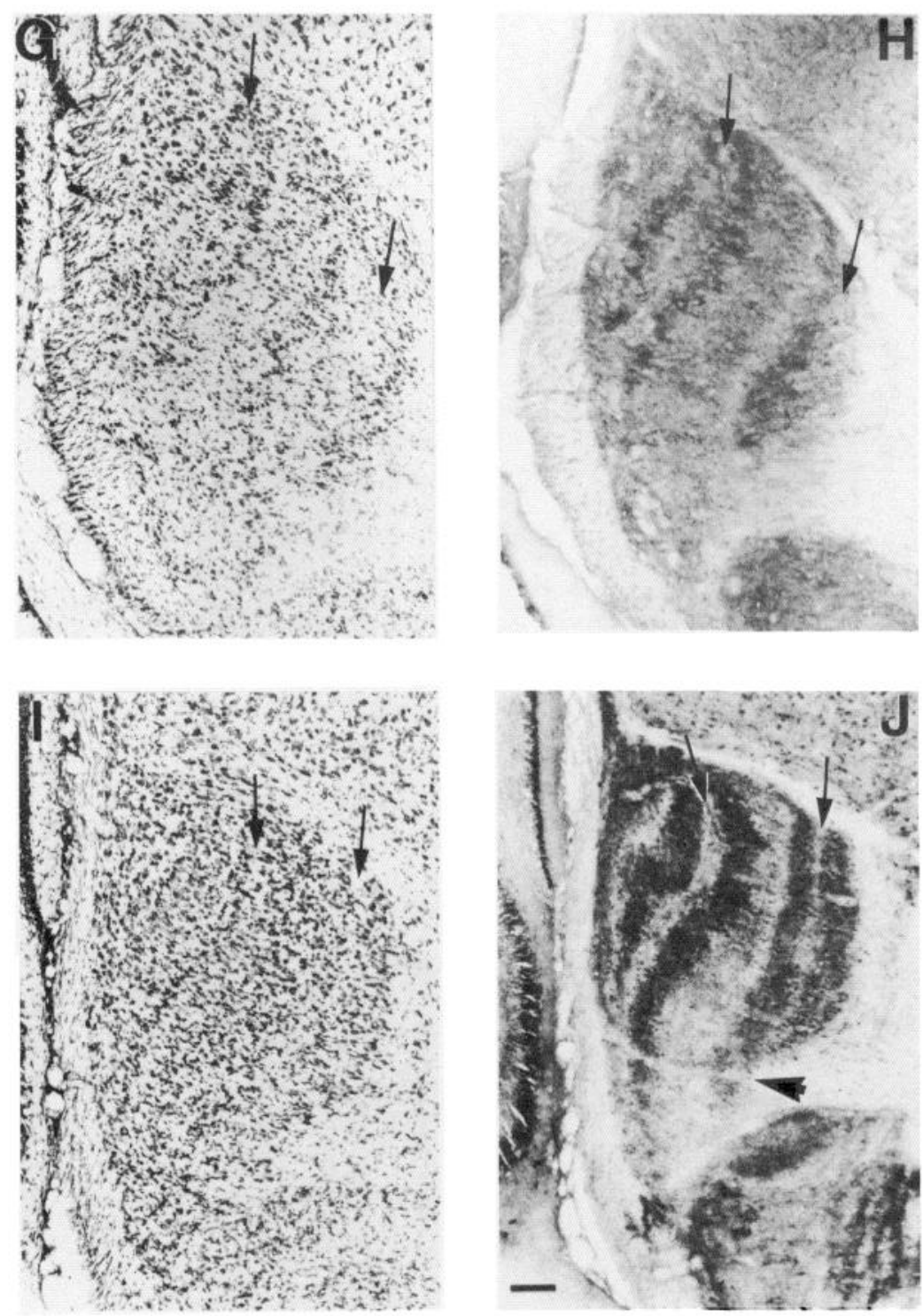

Figure 5. Continued.

suggest an obvious rearrangement or a loss of retinal projections to the LGN. However, the projecton pattern of retinal afferents is more similar to that of a P0 tree shrew than to that of a P14 tree shrew. Specifically, retinal afferents to layers 1 and 2 are more fused, and label in layers 3 and 6 is lighter than that of the control. The immature appearance of the retinal projections parallels the effects seen on the nucleus itself. However, from the slight differences in the density of label and spacing of projections, we cannot be certain that the immature appearancce of the retinal afferents is not simply a reflection of the small size of the nucleus.

In contrast to the above result, in one case in which one eye was injected for 2 weeks with TTX and that same eye was injected with WGA-HRP, the pattern of projections from the treated eye is broader than normal (see Fig. 13). In this case, the layers innervated by the right TTX-injected eye (i.e., 1 and 5 in Fig. 13, $B, D$, and 2, 3, 4, and 6 in Fig. 13, $A, C$, ) are markedly affected in terms of size and stain intensity in both Nissl and CO material; the TTX-affected layers are clearly less well-developed. Nevertheless, the retinal afferents of the TTX- injected eye (Fig. $13 F$, ipsilateral, and Fig. $13 E$, contralateral) are broader and extend beyond laminar borders, as defined by the Nissl- or CO-staining patterns. This result is particularly evident for input to layer 1 , which extends well into layer 2 (compare Fig. 13, $D$ with $F$ ). From these data alone we cannot determine whether the retinal fibers from the TTX-injected eye actually share the same territory with those from the other eye, although it seems reasonable to assume that they do, since the alternative would require that the normal fibers occupy less space within their home layer. In any case, the result is puzzling in light of the relatively normal (albeit immature) pattern of retinal projections in cases with bilateral TTX injections, and in light of the results we will consider next concerning cases in which the uninjected eye was removed on P0.

\section{Combined effects of TTX and enucleation}

Our results suggest that combining unilateral enucleation with unilateral TTX treatment is not equivalent to a bilateral enucleation. Instead, the resulting nucleus appears similar to that of a normal animal that was raised with one eye from birth 
Figure 6. Soma size distributions treatments on combined sets of LGN layers 1 and 2 and 4 and 5 , as well as for individual LGN layers 3 and 6 . As noted in Table $3 \mathrm{~A}$, all LGN layers innervated by TTX-injected eyes showed significantly smaller cells compared to matched sets of control innervated layers (mean soma sizes and statistical comparisons are shown in Table $3 \mathrm{~A}$ ). In all cases, the cells in the layers or sets of layers in bilateral TTX cases are much smaller; further, the mean differences between bilateral TTX and control cases accounted for $27-53 \%$ of the variance ventions as in Figure 4. showing the effects of bilateral TTX between these distributions. Other con-
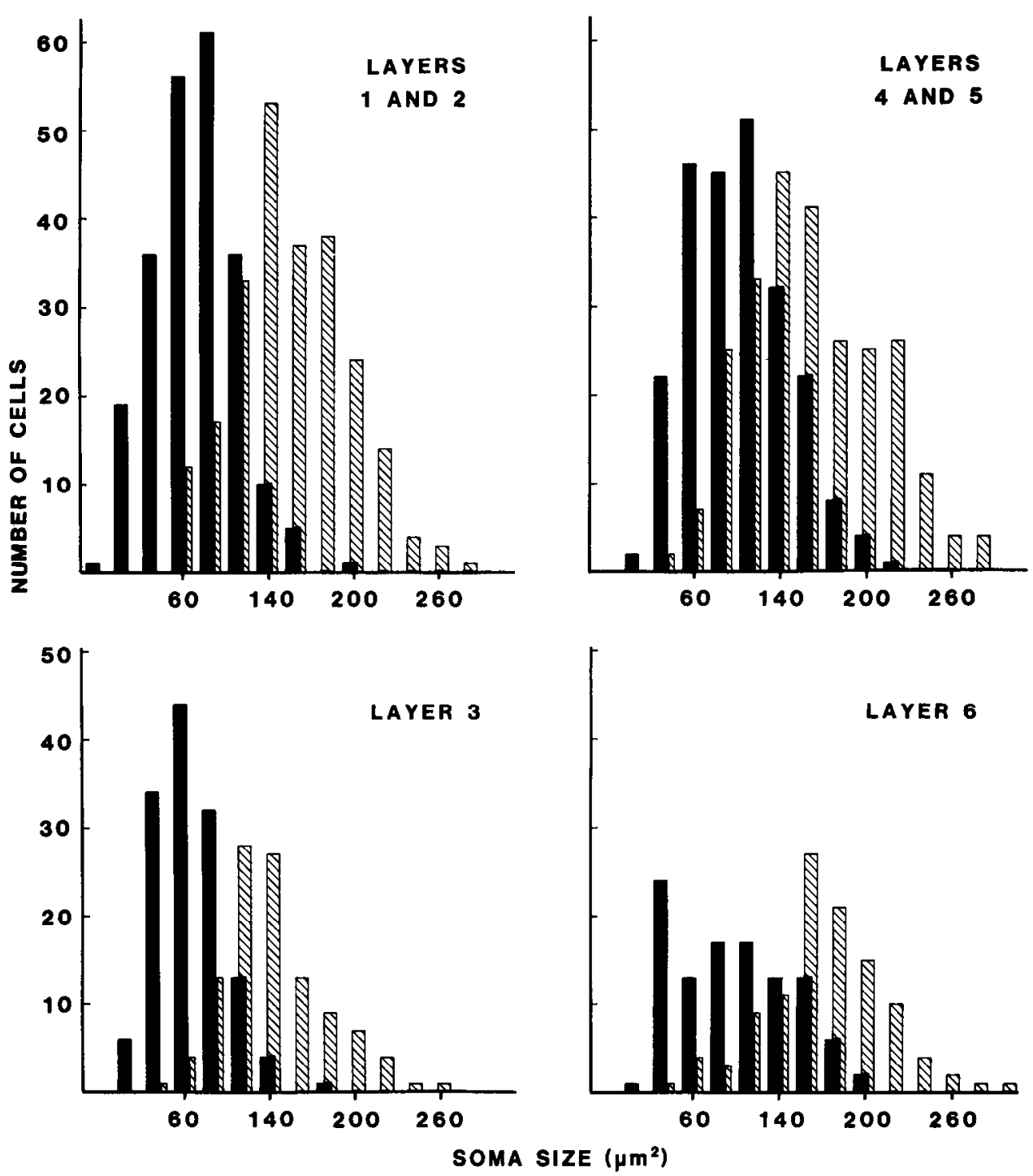

(Casagrande and Brunso-Bechtold, 1985). Figure 14 shows representative results from one of these cases. Ipsilateral to the TTX-injected eye (Fig. 14, B, D), 2 darkly stained layers are evident in both the Nissl and $\mathrm{CO}$ sections. Contralateral to the TTX-injected eye (Fig. 14, $A, C$ ), the remaining layers appear highlighted, although laminar differentiation in Nissl stain is less mature than that of an animal of the same age, monocularly enucleated at birth. Note also that in the $\mathrm{CO}$-stained section contralateral to the TTX-injected eye (Fig. 14C), layer 3 is more lightly stained than layers 2 and 4 , as is typical in normal tree shrews. These results again suggest that ganglion cell action potential activity is not essential for the differential pattern of laminar CO staining that is normally present. These data also suggest 2 important additional conclusions about the effect of TTX blockade. First, one eye by itself, with or without activity, is capable of producing recognizable laminar patterns both ipsilaterally and contralaterally. Second, the portions of the nucleus innervated by the silenced eye appear to develop a larger than normal territory, much as they do when the one eye that remains has normal action potential activity.
Figure 14, $E, F$ shows the pattern of retinal projections from the remaining TTX-injected eye in the same enucleated case shown in Fig. 14, $A-D$. This pattern of retinal projections is very similar to that from one eye in adult animals enucleated at birth (Casagrande and Brunso-Bechtold, 1985; Casagrande and Condo, 1987). In both cases, the number of retinal afferent laminar bands present is appropriate for one eye. However, input to layers 1 and 2 as well as 4 and 5 appears broader both ipsilateral (Fig. 14F) and contralateral (Fig 14E) to the remaining eye than is the case when 2 eyes are present. This observation suggests that either cells in the neighboring uninnervated layer move toward the site of innervation, forming larger-than-normal layers innervated by one eye, or more cells survive in the remaining innervated layers (see also Casagrande and Condo, 1988).

\section{Effects of APB on cellular lamination and metabolic activity}

Unlike what one might expect on the basis of the TTX results just described, daily injections of APB into one or both eyes, regardless of dose (see Methods), do not appear to selectively 

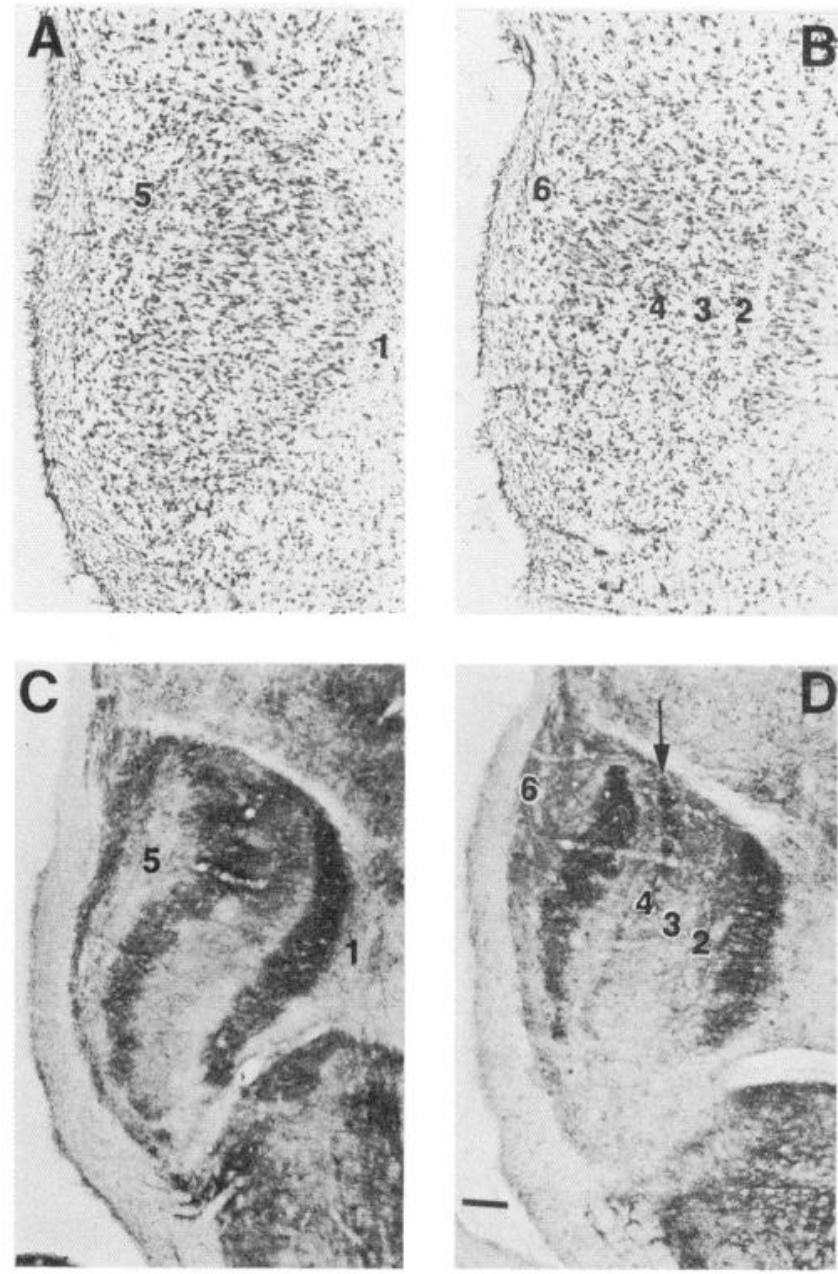

Figure 7. Nissl- $(A, B)$ and CO- $(C, D)$ stained horizontal sections through the LGN of case 85-80, in which the left eye was injected with TTX until P16. $A$ and $C$ are ipsilateral, and $B$ and $D$ are contralateral to the injected eye. Posterior is towards the top and lateral towards the left of each photomicrograph. The monocular segment of the nucleus lies anterior to ipsilaterally innervated layers 1 and 5 and is easily differentiated from the binocular segment in $D$, where it receives input from the TTX-injected eye. Numerals indicate the deprived layers. Note that the layers innervated by the TTX eye are much narrower, contain smaller cells, and are lighter-staining in both Nissl and CO sections. Moreover, the nucleus itself looks less well-differentiated than normal, especially in Nissl stain. Note also that all layers innervated by the TTX eye are not equally affected by the treatment. The arrow in $D$ shows that one leaflet of deprived layer 4 still stains darkly in $\mathrm{CO}$, indicative of higher metabolic activity. See text for details. Scale bar, $0.1 \mathrm{~mm}$.

affect the development of layers 1 and 2 (which contain exclusively ON-center cells) or the interlaminar space between them. However, the entire nucleus does, in some cases, appear immature. Figure 15 compares LGN laminar development in 3 P6/7 animals: case 84-14, a control case (Fig. 15, $A, B$ ), case 84-16, in which both eyes were injected with APB (Fig. 15, C, $D$ ), and case 84-15, where the right eye was injected with APB (Fig. 15, E, F). In the latter case, the sections are shown ipsilateral to the eye injection (i.e., layers 1 and 5 receive input from the APB-treated eye). As can be seen in these examples, the layer 1-2 and 4-5 interlaminar spaces that are normally present at this age (Fig. 15A) are well-developed in the APB cases (Fig. 15, $C, E$ ). Additionally, we find that layers 1 and 2 show normal CO activity (compare Fig. 15, $B, D$ and $F$ ). Finally,
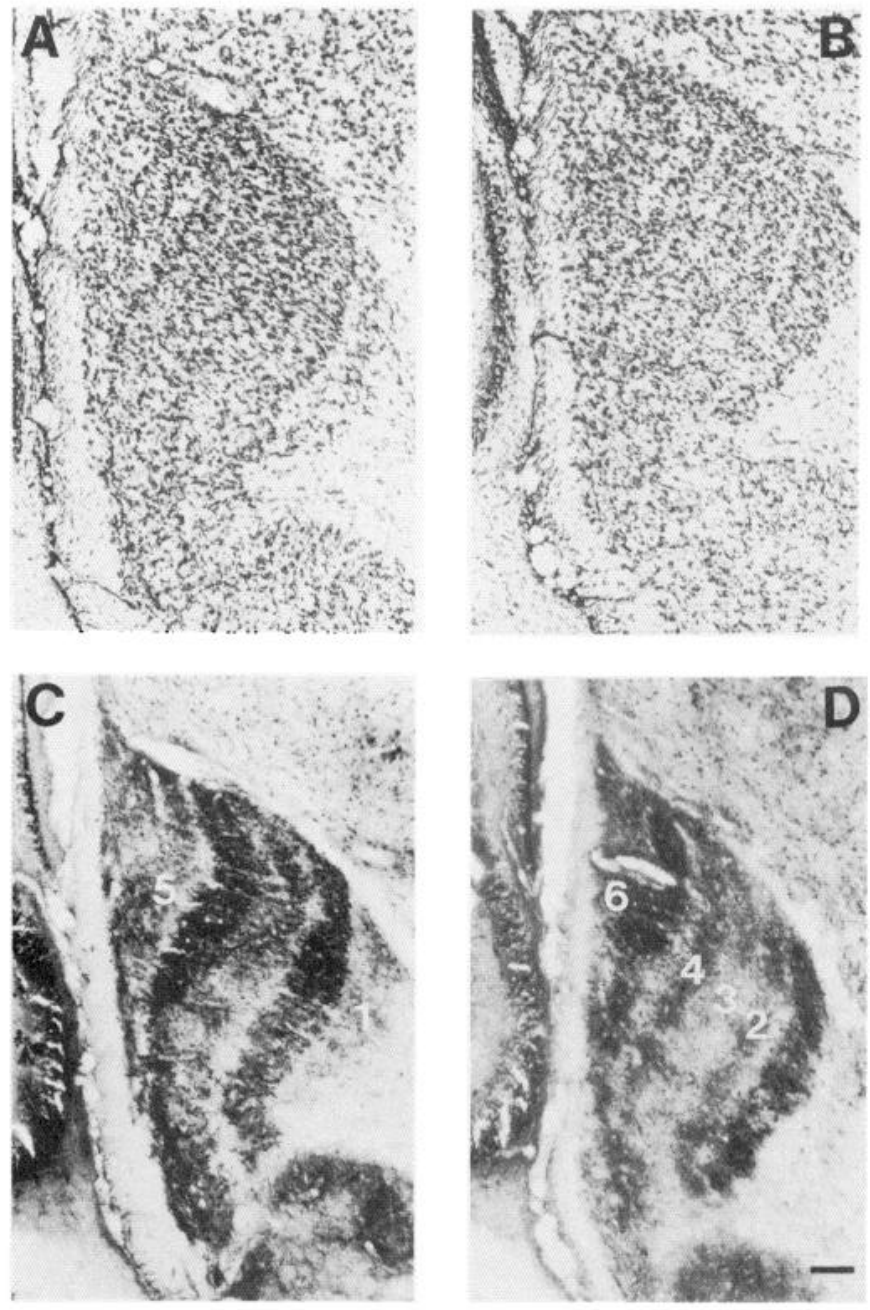

Figure 8. Nissl- $(A, B)$ and CO- $(C, D)$ stained horizontal sections through the LGN of case $85-71$, in which the left eye was injected with TTX until P15. Posterior towards the top and lateral towards the left of each photomicrograph. $A$ and $C$ are ipsilateral, and $B$ and $D$ are contralateral to the eye injection. Numerals indicate the deprived layers in the CO sections in $C$ and $D$. See text for details. Scale bar, $0.1 \mathrm{~mm}$.

in cases that survived daily APB injections until P14, LGN lamination appears robust and is no different from that of a normal P14 tree shrew (see Fig. 16).

\section{Discussion}

The main objective of this study was to test whether LGN cell layer formation, defined by the formation of cell-sparse interlaminar spaces (i.e., cytoarchitectonically defined lamination), is dependent on the presence of action potential activity in retinogeniculate axons. As mentioned in the introduction, previous studies have shown that formation of cell layers, as defined here, is prevented, and maturation of the surviving LGN cells is slowed by early eye removal. Surviving cells in the LGN, however, eventually develop the cytological characteristics typical of their layer, as well as topographic connections with cortex (Brunso-Bechtold and Casagrande, 1981; Brunso-Bechtold et al., 1983). Since various manipulations of patterns of optic nerve activity have profound effects on the growth and function of LGN cells in many mammals, including tree shrews (Casagrande et al., 1978; see also Sherman and Spear, 1982), we concluded 

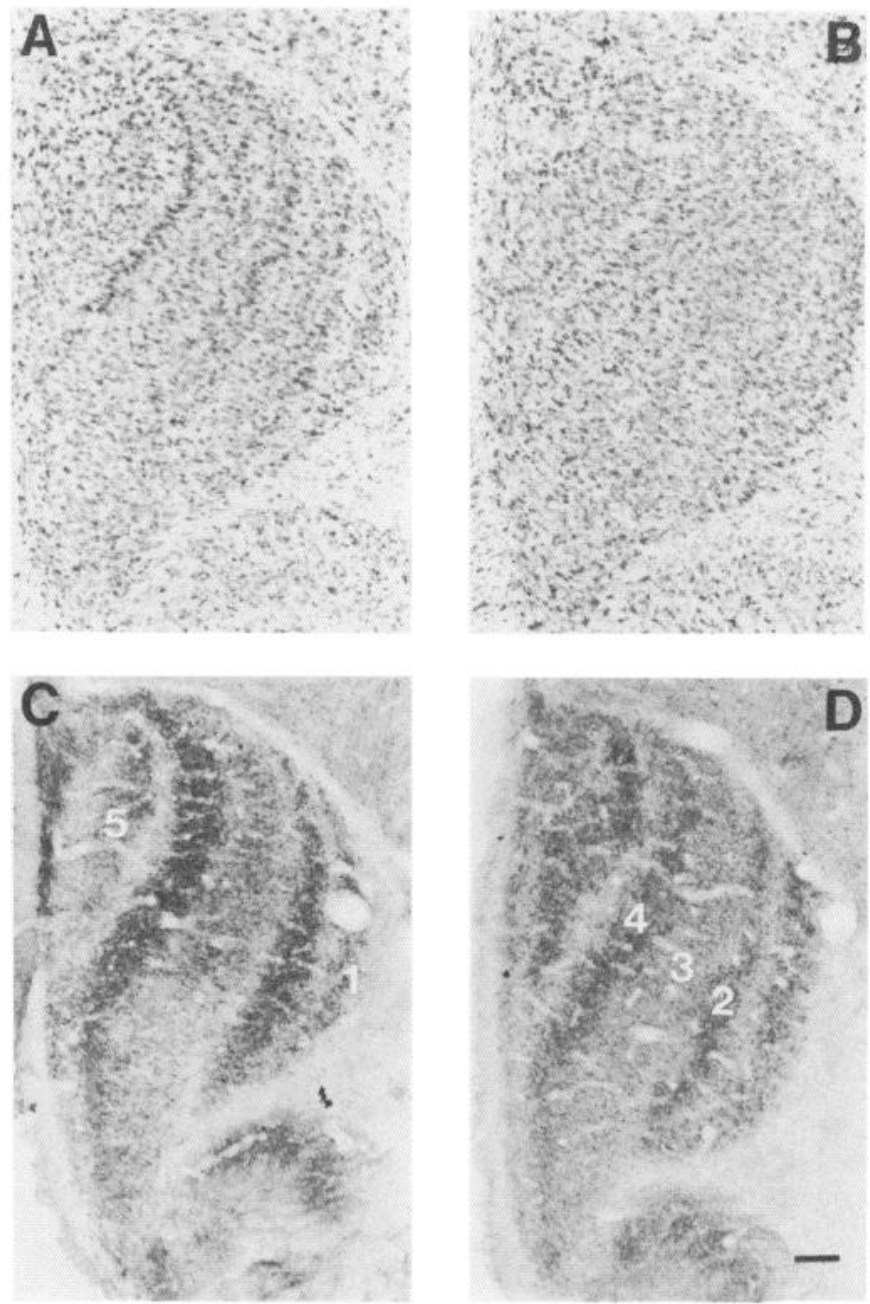

Figure 9. Nissl- $(A, B)$ and $C O-(C, D)$ stained horizontal sections through the LGN of case $85-115$, in which the left eye was injected with TTX until P13. Posterior is towards the top and lateral towards the left of each photomicrograph. $A$ and $C$ are ipsilateral, and $B$ and $D$ are contralateral to the eye injection. Numerals indicate the deprived layers in the $C O$ sections in $C$ and $D$. See text for details. Scale bar, 0.1 $\mathrm{mm}$.

that optic nerve activity could play a role in the development of LGN cell layers. The present study further examined this issue in detail, and the results clearly show that incipient interlaminar spaces can develop in the absence of such activity, but that retinal activity is an important factor in supporting the normal rate of LGN laminar development.

In the discussion that follows, we consider the implications of our findings in light of previous work. We then consider the general implications of the results in relation to a possible model of LGN laminar development.

\section{Manipulations of light-evoked activity with APB}

Attempts to manipulate light-evoked or spontaneous activity within ON-center retinal ganglion or LGN cells with APB did not selectively affect the relative development of LGN ONcenter layers. These results are difficult to interpret. In adult rabbits, macaque monkeys, cats, tree shrews, and neonatal kittens, there is clear evidence that intraocular injections of APB can block or attenuate light-evoked $\mathrm{ON}$-center responses from ON-center or ON-/OFF-center cells (Horton, 1981; Sandell and
Schiller, 1982; Schiller, 1982 a, b; Knapp and Mistler, 1983; Smith et al., 1985; T.T. Norton, personal communication). It has been argued that this effect results from APB's ability to mimic the effects of the presumed endogenous photoreceptor transmitter, glutamate (Slaughter and Miller, 1981). Thus, if we had been successful in manipulating the activity of the ONcenter channel during early development, we might have expected to find a selective slowing of the differentiation of LGN layers 1 and 2, which contain ON-center cells (Conway and Schiller, 1983), much as we found more generally following TTX injections. Instead, we found evidence only for a slight overall slowing of LGN laminar differentiation. The lack of a selective effect could be interpreted in several ways. First, since the retina is very immature, it may be that the activity of ON-center ganglion cells is not dependent on the activity of the even less mature ON-bipolar cells and receptor cells. Indeed, recent evidence suggests that $\mathrm{ON}$ - and OFF-center cell channels in the tree shrew retina are relatively undifferentiated in CO-stained material until after the differentiation of LGN cell layers (Lachica et al., 1987). Second the dose schedules and amounts we chose may not have been correct in spite of the fact that they were appropriate for adults. Finally, it is reasonable to imagine that APB's main effect would be on light-evoked activity, and not on spontaneous activity, although Knapp and Mistler (1983) clearly show that APB also affects levels of spontaneous activity in the ON-center LGN cells of adult rabbits. The slight slowing of overall LGN development with APB injections resembles, in mild form, the results of completely blocking action potential activity, suggesting that APB may have some general effect on retinal ganglion cell action potential activity.

\section{$L G N$ development in the absence of retinal action potentials}

Our results show that interlaminar spaces will begin to form between cell layers even when retinal action potentials are completely blocked in both eyes from birth until 2 weeks of age. This would suggest that factors other than activity are responsible for cytoarchitecturally defined cell layer formation. Before we consider the implication of this finding, it is essential that we consider the possibility that, as with the APB injections, the TTX doses we employed were not sufficient to block activity during the period we examined. We would argue that this is not the case on 2 grounds. First, the lowest doses we employed were sufficient to block cortical VEP activity in adults. Second, and more important, our VEP measures on infant tree shrews suggested that our lowest doses were sufficient to block VEP activity for more than $2 \mathrm{~d}$. Dubin et al. (1986) have reported that cortical VEP activity is an extremely sensitive measure of recovery of ganglion cells from the effects of TTX and can readily be recorded in kitten cortex when only a few ganglion cells can be visually driven and no spontaneous activity can be detected. Nevertheless, since we were not able to obtain VEPs from the very youngest tree shrews (P0-P4), presumably because of the immaturity of the retina, it remains possible that the TTX doses did not completely block activity at these earliest ages. Nevertheless, this would not affect the overall outcome, since cell layers were not differentiated in bilaterally treated TTX animals of less than $4 \mathrm{~d}$ of age.

Given that interlaminar space formation in the LGN (i.e., cellular lamination defined cytoarchitectonically) can be initiated in the absence of retinal ganglion cell action potential activity, but not in the absence of retinal ganglion cell axons in tree shrews (Brunso-Bechtold and Casagrande, 1981), it is likely 

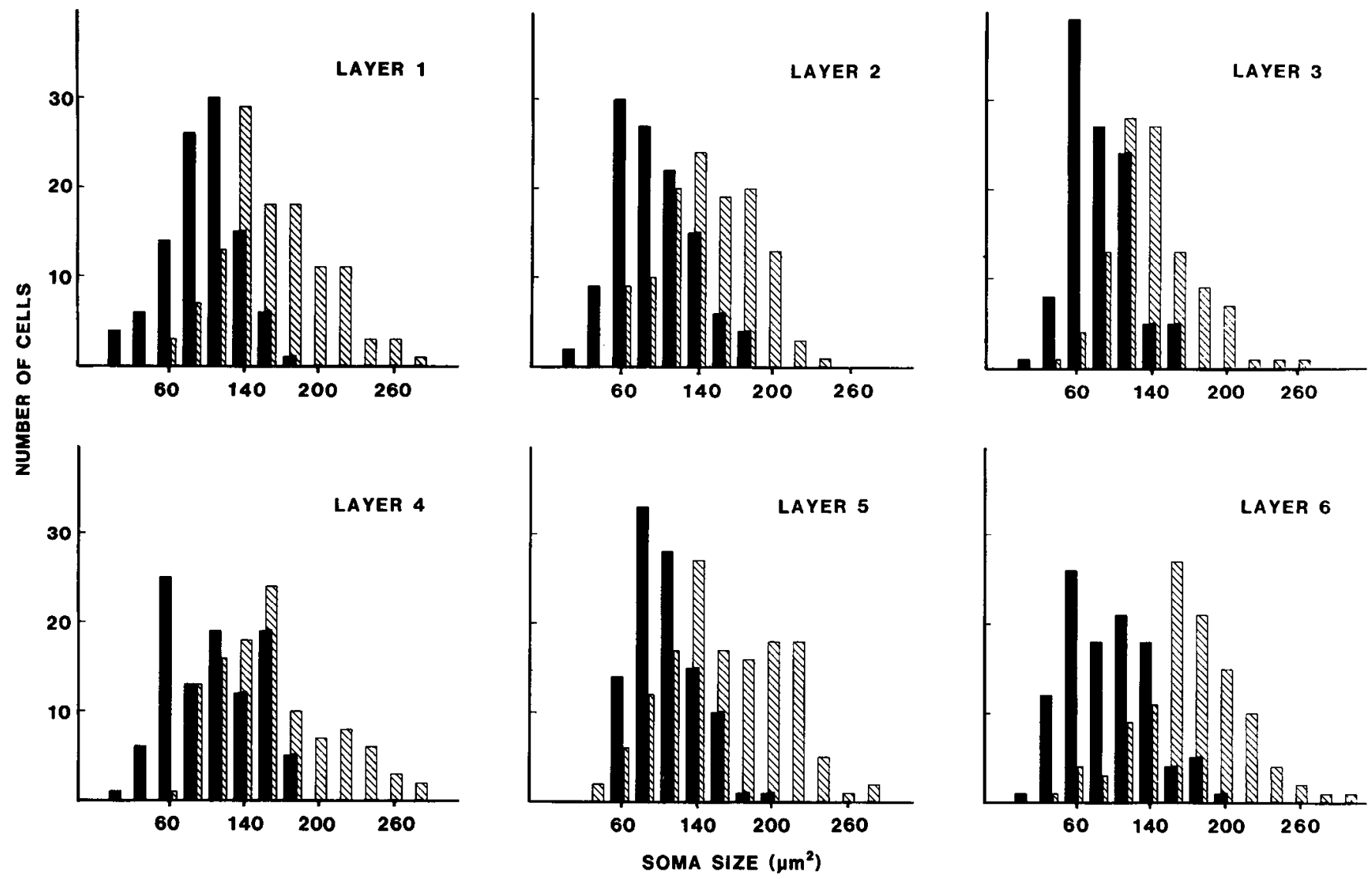

Figure 10. Soma size distributions of LGN layers innervated by unilateral TTX eye injections (filled) compared to layers innervated by control injected eyes (cross-hatched). As noted here and summarized in Table 3B, all TTX-innervated layers show significantly smaller cell sizes compared to layers innervated by control injected eyes. Also, the overall effect of bilateral TTX treatments was more dramatic than that of unilateral TTX treatments (compare with Fig. 6; see also Table 3). Indeed, inspection of the ranges of omega square values in Table 3 suggests that unilateral TTX injections result in more variable, albeit statistically significant, differences.

that the silent retinogeniculate axon terminals release trophic factors that stimulate the growth and "movement," and/or maintain the select viability of LGN cells in such a manner as to produce spaces between cell layers (see below). The main point here is that retinal ganglion cell activity per se does not appear to be involved in initiating the process of cell layer formation defined cytoarchitectonically. By this, we do not mean to imply that activity in ganglion cells is not important to the

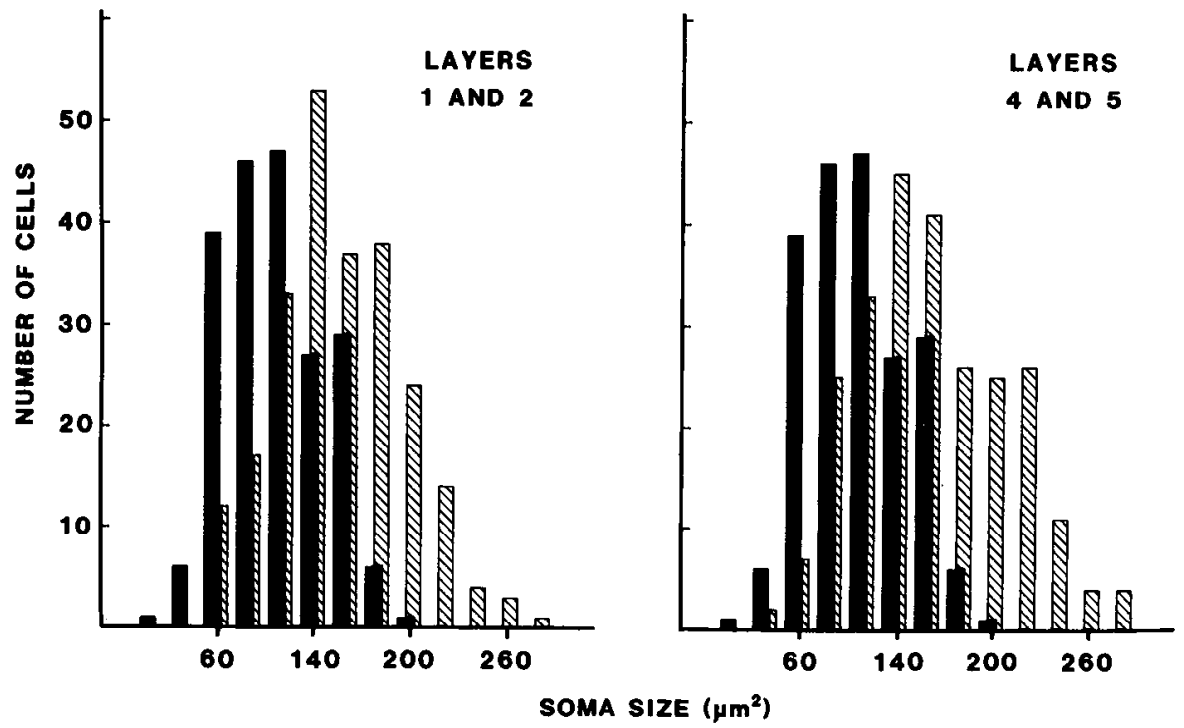

Figure 11. Soma size distributions for matched sets of $L G N$ layers innervated by TTX-injected eyes compared to matched sets of LGN layers innervated by control injected eyes. As noted here and summarized in Table $3 \mathrm{C}$, the overall TTX effect on cell size is more dramatic in layers 1 and 2 than in layers 4 and 5. Other conventions as in Figure 4. Sec text for details. 

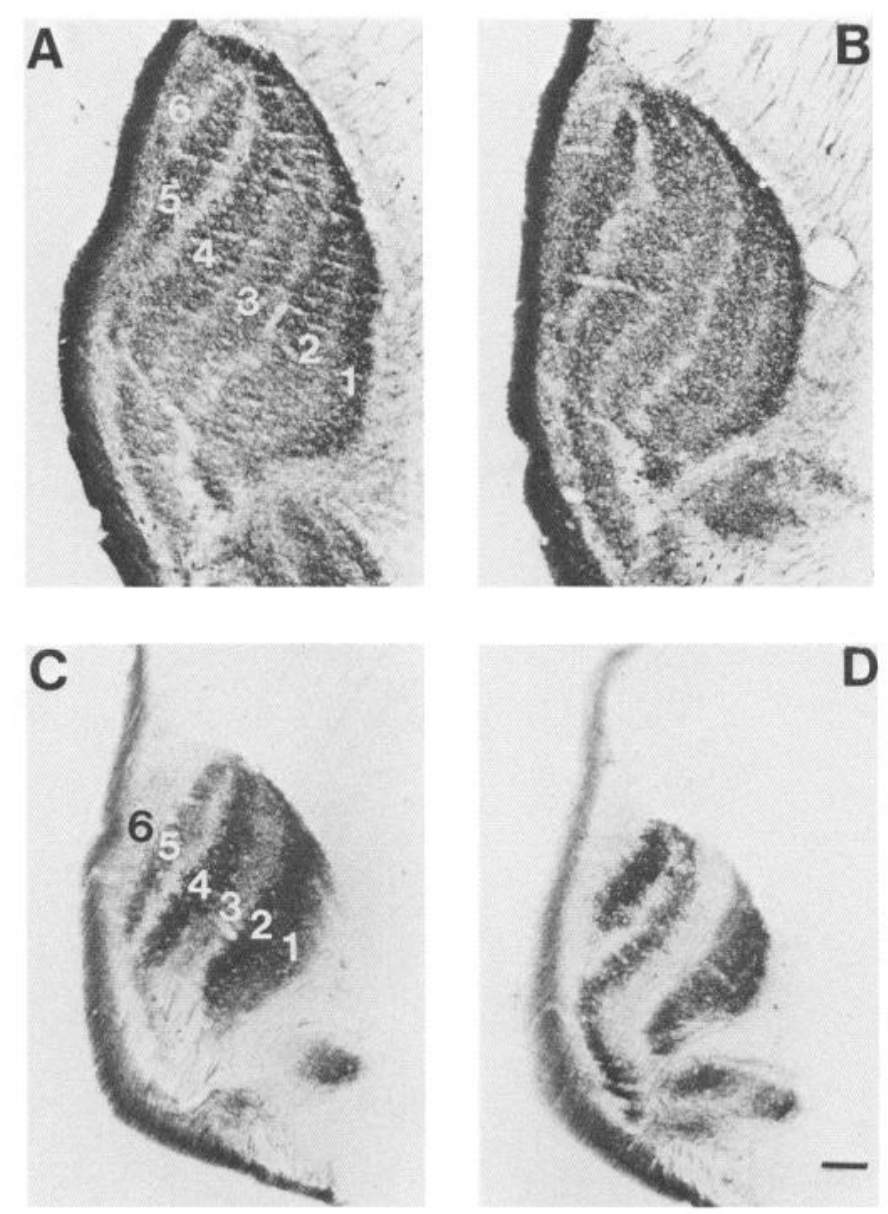

Figure 12. Horizontal sections (one from each hemisphere) through the LGNs of a normal P14 tree shrew (86-11) that received bilateral WGA-HRP eye injections $1 \mathrm{~d}$ prior to being killed $(A, B)$, and a tree shrew (85-191) that received bilateral TTX eye injections until P14 and bilateral WGA-HRP eye injections $1 \mathrm{~d}$ prior to death $(C, D)$. All sections were reacted with tetramethylbayzidine to demonstrate the presence of the HRP. Posterior is towards the top and lateral towards the left of each photomicrograph. At P14, retinal afferents from the 2 eyes show a normal adultlike pattern of segregation that, with one exception, matches the boundaries of the cell layers. The exception involves the retinal input to layers 1 and 2 , which is barely separated and extends into the interlaminar spaces. Compare the space between retinal afferents to layers 1 and 2 with those to layers 4 and 5 in $A$ and $B$ (see also Fig. $3 A$ ). Following TTX treatment $(C, D)$, afferents remain segregated but the retinal input to the nucleus resembles that seen at P0, where the input to layers 3 and 6 is lighter, and the separation between retinal afferents bands is slightly less than for more mature animals.

sequence of steps that precedes normal LGN cell layer development. Recent observations of Shatz and Stryker (1986) clearly demonstrate that segregation of retinogeniculate axons depends upon neuronal activity: normal segregation is prevented in fetal kitten embryos infused with TTX. Since segregation of retinogeniculate axons within the LGN precedes cell layer development, and since interlaminar spaces never develop in the LGN at locations where retinal axons are not also segregated (although the opposite can occur), it follows that very early blockade of neural activity, if continued into the period of normal LGN cell layer formation, will prevent such formation by preventing retinogeniculate axonal segregation. Obviously, this scenario remains to be proven experimentally.

In the present experiments, TTX blockade was initiated at birth, when retinal ganglion cell axons are already segregated into laminar bands within the undifferentiated LGN (Rager et al., 1980; Brunso-Bechtold and Casagrande, 1982; Casagrande and Brunso-Bechtold, 1985). If, as a number of investigators have argued, activity is important in the segregation of retinal axons and for the stability of synaptic relations between preand postsynaptic elements (Reh and Constantine-Paton, 1985; Schmidt, 1985), then one might predict that silencing action potential activity would remove important feedback necessary to keep axons in a segregated state, and allow them to return to their earlier prenatal overlapping state. In fact, Reh and Constantine-Paton (1985) have already shown that silencing activity in the optic nerves of 3-eyed frogs, after axons have segregated into ocular dominance bands within the optic tectum, results in "debanding." In other words, silencing activity appears to release optic axons from the constraints of the ocular dominance bands and allows the axons from each eye to move into overlapping territories. Our results show that this situation does not apply to the already segregated retinogeniculate axons of the tree shrew, which remain segregated even after 2 weeks of bilateral TTX treatment. Apparently, optic nerve activity is required only for the process, not for the maintenance of retinogeniculate axonal segregation. It may be that the activity-dependent segregation itself results not from movement of axons, as must happen in the optic tectum of frogs, but rather from a loss of inappropriately located axonal collaterals and/or the loss of a select subset of ganglion cells whose axon terminals lie within an inappropriate area of the LGN (Constantine-Paton and Law, 1978; Constantine-Paton, 1982; Sretavan and Shatz, 1984, 1986; Reh and Constantine-Paton, 1985; Rakic, 1986). If that is the case, then it is easy to see why neural activity would not necessarily be required to maintain retinogeniculate axons within a segregated state.

A final aspect of LGN laminar development in tree shrews that does not appear to be affected by silencing optic nerve activity is the expression of cytologic and metabolic differences between the layers. At birth, the morphology of individual LGN cells in Nissl-stained material from tree shrews appears relatively uniform, and metabolic activity, as revealed by the $\mathrm{CO}$ mitochondrial stain, appears relatively homogenous. Following bilateral TTX treatment for 2 weeks, cytological differences (albeit cells are less mature than normal) are apparent between cells in individual developing layers. Further, CO-stained material clearly reveals the differential pattern of relatively lightand dark-staining layers characteristic of adults (Norton and Wong-Riley, 1984). Thus, optic nerve activity is not required for the expression of these differences. The lack of effect on LGN cytology is less surprising, since removal of both eyes at birth does not prevent the expression of some morphological differences between cells that would normally lie in different layers (Brunso-Bechtold and Casagrande, 1981). It may also be the case that the presence of retinogeniculate axons is unnecessary for the expression of staining differences revealed by $\mathrm{CO}$, although we have not yet examined the $\mathrm{CO}$ staining pattern of animals bilaterally enucleated at birth.

Since cytological laminar differences can develop in cases where LGN cells have never been contacted by retinal axons (Guillery et al., 1985), it is reasonable to propose that such differences reflect an expression of intrinsic differences between LGN cells belonging to different layers. It may also be the case that the differential CO laminar staining is another reflection of intrinsic regional differences within the LGN. The importance of this point will become more apparent when we consider what mech- 

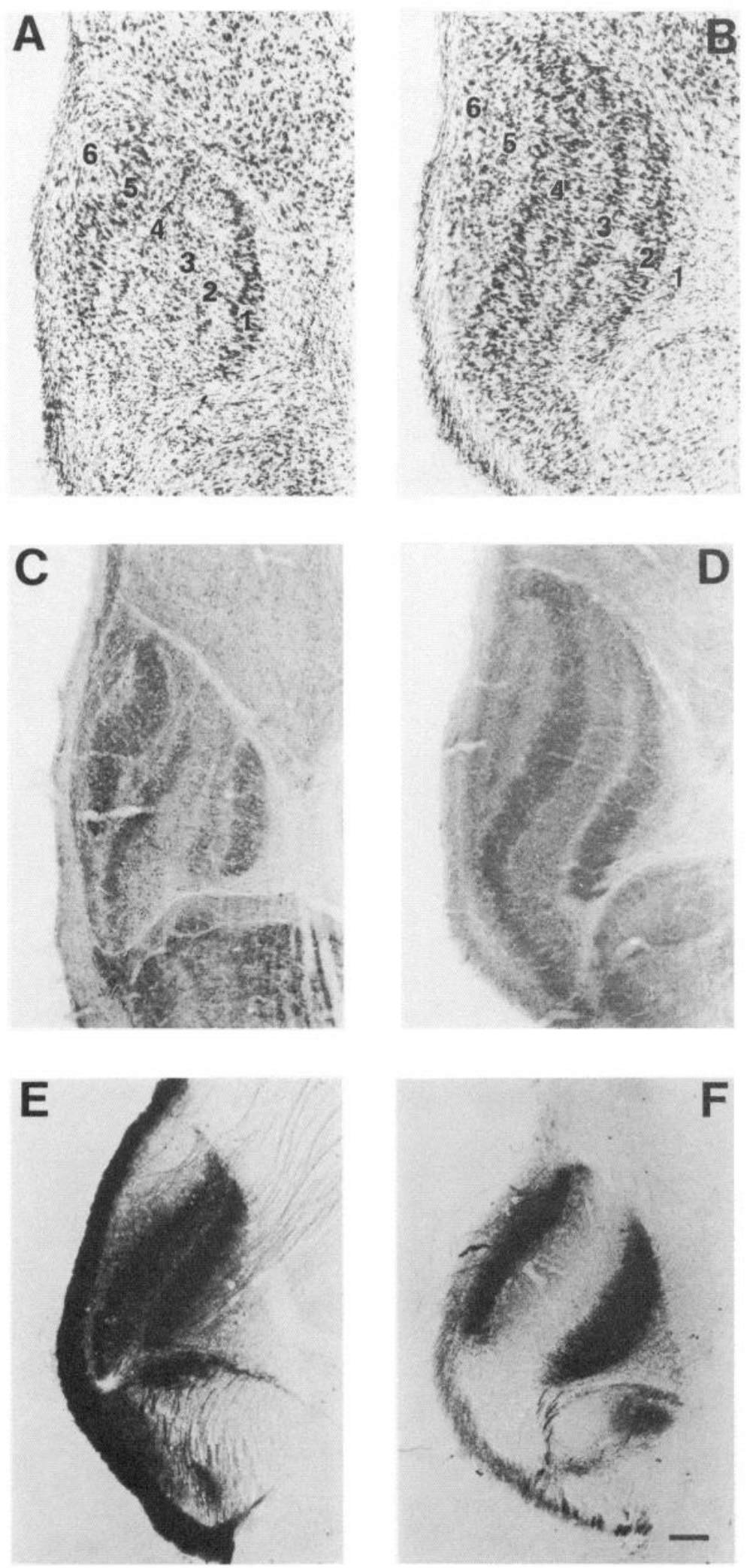

Figure 13. Horizontal sections through the LGN of a tree shrew (85-194) that received TTX injections into the right eye, had a WGA-HRP injection in the same eye on P14, and survived until P15. Posterior is towards the top and lateral towards the left of each photomicrograph. $A, B$, Nissl-stained sections contralateral and ipsilateral to the TTX eye injection, respectively. Note that the layers innervated by the TTX-treated eye $(2,3,4$, and 6 in $A$, and $I$ and 5 in $B)$ are narrower and more lightly stained. $C, D, \mathrm{CO}$-stained sections contralateral and ipsilateral to the TTX eye injection, respectively. Again, the layers innervated by the TTX-treated eye are narrower, and, in the case of layers 1 and 2 , are also lighter-staining. $E, F$, Pattern of retinal input from the TTX-treated eye contralateral and ipsilateral to that eye, respectively. Note that the input to the TTX-treated layers is very robust and extends into adjacent layers that presumably receive normal innervation. This is especially evident ipsilateral to the TTX-treated eye, where input to layer $1(F)$ clearly extends into layer 2; compare with $B$ and $\mathrm{D}$. $\mathrm{Nu}$ merals in $A$ and $B$ indicate the LGN layers. See text for details. Scale bar, $0.1 \mathrm{~mm}$.

anisms could be involved in LGN cell layer formation.

So far, we have only considered aspects of LGN development that proceed relatively normally in the absence of optic nerve activity. Our results also clearly demonstrate that complete blockade of sodium-dependent action potential activity by in- jection of TTX in both eyes disrupts several aspects of normal LGN laminar development.

The most obvious effect of such treatment is that the LGN is immature by several criteria. The segregation between retinogeniculate projections is narrow, the relative size of the nucleus 

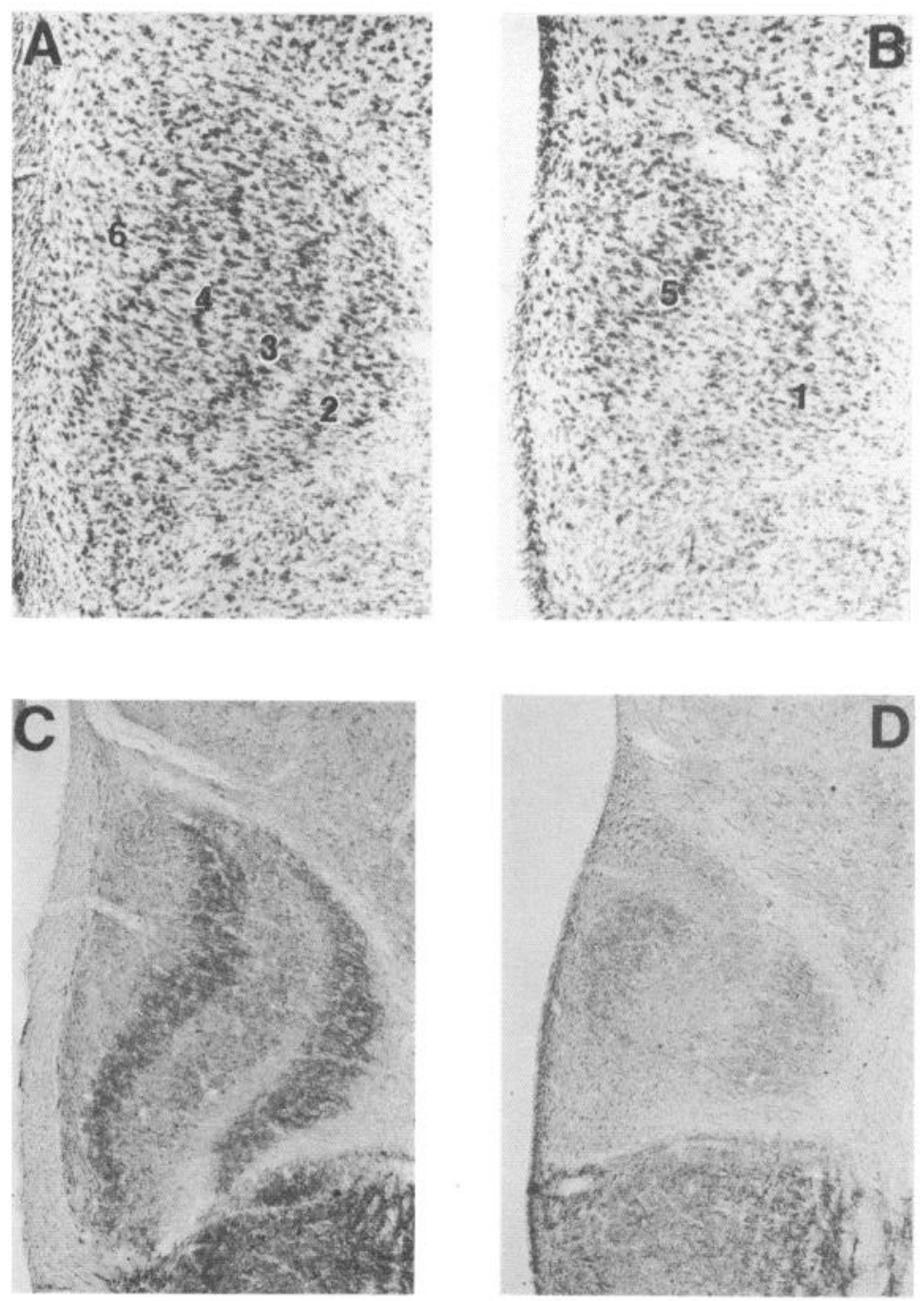

Figure 14. Horizontal sections through the LGN of a tree shrew (85-31) that had its left eye removed at $\mathrm{PO}$ and its right eye injected with TTX until P16. This animal also received an injection of WGA-HRP into the TTX-treated eye on P15 and was killed on P16. Posterior is towards the top and lateral towards the left of each photomicrograph. $A, B$, Nissl-stained sections contralateral and ipsilateral to the TTX eye injection, respectively. Note that the normal complement of 4 contralateral $(2,3,4,6)$ and 2 ipsilateral $(1,5)$ layers are produced by the TTX-treated eye, although the layers themselves are broader than normal and their position distorted, especially ipsilaterally. This result is similar to that seen following a P0 unilateral enucleation without subsequent TTX treatment, except that following enucleation alone, lamination appears better differentiated. $C, D$, $\mathrm{CO}$-stained sections contralateral and ipsilateral to the TTX eye injection, respectively. The CO laminar pattern appears to match the distribution of cells shown in $A$ and $B . E, F$, Pattern of retinal input to the nucleus contralateral and ipsilateral to the TTX-labeled eye, respectively. The retinal projections match the Nissl and $\mathrm{CO}$ laminar patterns. Numerals indicate the innervated LGN layers. See text for details. Scale bar, $0.1 \mathrm{~mm}$.
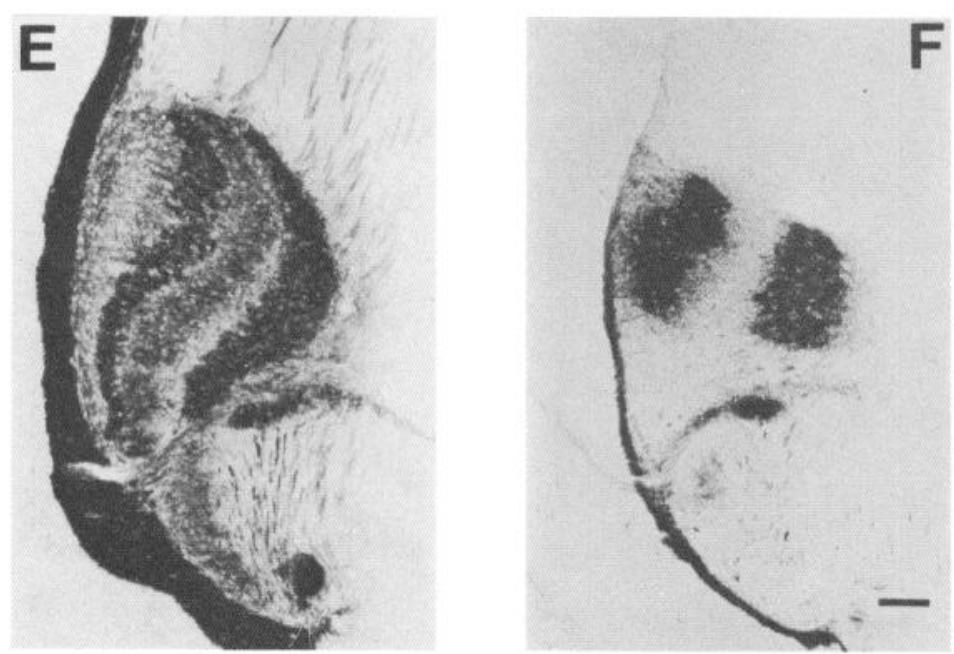

and of the cells themselves is smaller, and the morphological structure of the cells and extent of interlaminar spaces all appear retarded in their development. In addition, some aspects of LGN development are more affected by the lack of neural activity than others. Thus, the size of cells in layers 1 and 2, and the development of the interlaminar space between these layers is smaller following TTX treatment than is the case for the remainder of the nucleus.

At present, it is unclear how blockade of optic nerve activity influences the development of the LGN. Several (not mutually 

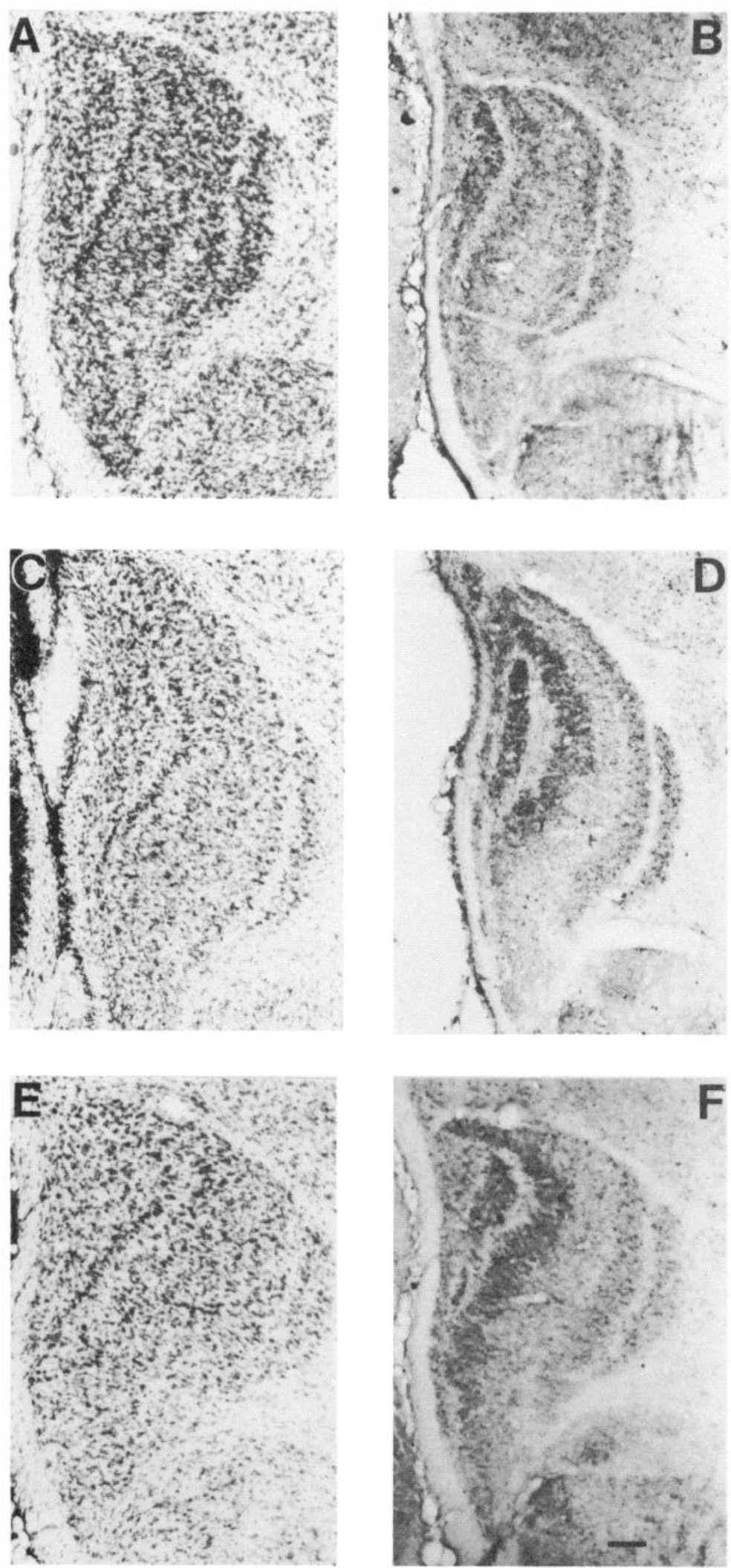

Figure 15. Nissl- $(A, C, E)$ and CO$(B, D, F)$ stained horizontal sections through the LGNs of 3 tree shrews that survived until P6/7. Posterior is towards the top and lateral towards the left of each photomicrograph. $A, B$, Sections from a control case (84-14) that received daily saline injections into the right eye. The sections shown are contralateral to the eye injection; ipsilateral sections look similar. $C, D$, Sections from a case (84-16) that received bilateral daily APB eye injections. Both sections are from the right LGN; the left LGN looked similar $E, F$, Sections from case 84-15, which received unilateral daily injections of APB into the right eye. The sections shown are contralateral to the injected eye; ipsilateral sections looked similar. Note that the space between layers 1 and 2 is welldeveloped in all 3 cases. See text for details. Scale bar, $0.1 \mathrm{~mm}$. exclusive) possibilities exist. First, the normal rate of growth of retinogeniculate axons and their targets could depend directly on the presence of an adequate amount of neural activity for stimulation of the processes involved. This possibility is partially supported by observations of the LGN of kittens raised with TTX blockade of ganglion cell activity. In such kittens, Kalil et al. $(1983,1986)$ showed that retinal terminals (RLP profiles) were smaller, made fewer synaptic contacts, and that those present were immature in morphology following 8 weeks of blockade of ganglion cell activity from birth. Also, Kupper- 


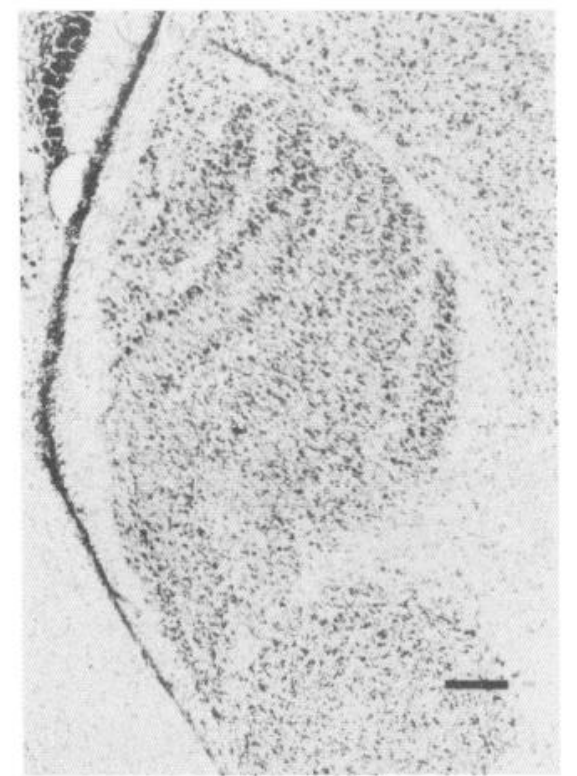

Figure 16. Nissl-stained horizontal section (posterior toward the top, lateral towards the left) through the LGN of a tree shrew (84-62) that received bilateral daily eye injections of APB until P14. The LGN is well-differentiated and not different from that of a normal P14 tree shrew. Compare with Figure 3. Scale bar, $0.1 \mathrm{~mm}$.

man and Kasamatsu (1983) reported that after only 1 week of monocular TTX treatment, deprived LGN cells in both 7-weekold kittens and adult cats were $30 \%$ smaller than their normal counterparts. This result, in addition to the results reported by Wong-Riley and Riley (1983), Wong-Riley (1979), and WongRiley and Carroll (1984), which demonstrated a decrease in CO activity in "deprived" LGN layers in cats and monkeys following monocular TTX treatment, supports the idea that the normal cellular metabolism of ganglion cells and LGN cells is dependent on action potential activity. A second, related possibility is that appropriate activity is essential to normal synapse formation and stabilization (Constantine-Paton, 1982; Fawcett and O'Leary, 1985; Schmidt, 1985; Schmidt and Tieman, 1985; Dubin et al., 1986), suggesting that ganglion cells and LGN cells require that this step be complete (to a certain point) before further differentiation takes place. Finally, the effect on activity could be secondary to the effect of TTX on the transport of specific glycoproteins and the terminal release of trophic factors (Edwards and Grafstein, 1983, 1984; Ricco and Matthews, 1983).
It is more difficult to interpret our results showing a differential TTX effect on the development of LGN layers 1 and 2. In normal adult tree shrews, layers 1 and 2 are unique in several ways. First, they receive exclusive retinal input from ON-center ganglion cells (Conway and Schiller, 1983; Holdefer and Norton, 1986). Second, unlike the other layers, there is a mismatch between the well-developed space between these layers and the poorly developed space between the retinal afferents terminating in these layers. This suggests that the spacing of cells into layers 1 and 2 is dependent on more than just the spacing of the retinal afferents. Finally, the layer 1 and 2 interlaminar space is unique in lacking input from the superior colliculus, an input that is normally prominent in all of the other interlaminar spaces within the nucleus (Casagrande, 1974; Fitzpatrick et al., 1980; Sutton and Brunso-Bechtold, 1985). Presumably, then, the differential effect of activity blockade on layers 1 and 2 reflects a selective effect on either ON-center ganglion cells or the pace of development of important sources of extraretinal innervation.

\section{LGN development following unilateral TTX blockade}

Our results show that the effects of unilateral TTX blockade are similar to, but more severe than, those of bilateral, or total, TTX blockade. In some cases (i.e., Fig. 7), inspection of the deprived layers suggests that, in addition to cell size changes, there is cell loss. This may be due to the combined disadvantages for the LGN cells of loss of appropriate levels of activity and, secondarily, loss of synaptic space in striate cortex due to unequal competitive interactions between geniculate cells innervated by the normal and "deprived" eyes (Casagrande et al., 1978).

Other than the asymmetry in LGN laminar development that results following unilateral TTX treatment, the only other notable difference between the unilateral and bilateral TTX treatment groups concerns the distribution of retinal projections. In the one case in which we labeled retinogeniculate projections from the TTX-treated eye following $14 \mathrm{~d}$ of TTX treatment, the projection from that eye extended beyond laminar boundaries, as defined by $\mathrm{CO}$ or Nissl stain. This would suggest that, unlike the relatively stable state of retinogeniculate projections following bilateral TTX treatment, the unilaterally silenced retinogeniculate axons are capable of extending processes into neighboring (presumably normally innervated) layers. Dubin et al. (1986) report that, unlike the case in normal kittens, numerous LGN cells are excited by both eyes following 2-3 months of unilateral TTX treatment from birth. Dubin et al. (1986) suggest that their results might be accounted for either by sprouting (of the sort we see in our material) or by maintenance of

Figure 17. One possible scenario of how layers may form in the LGN, based on evidence from our work. The drawings at the top indicate the cytoarchitectural appearance of the nucleus in horizontal section (posterior towards the top and lateral towards the left) at 3 ages: day of birth (P0); during the middle of cell layer formation (P3-P4); adult; and adult bilaterally enucleated at P0. The drawings at the bottom illustrate a hypothetical sequence of cellular development at these different stages for 2 adjacent layers. Vertical lines represent retinal afferent projections, and stippling represents extraretinal afferent projections at each stage of laminar development. At birth (P0), as represented in this model, retinal afferents have segregated into laminar bands over a cytologically immature nucleus, where no cytoarchitectonic differentiation is evident and cells have few dendrites. During the first postnatal week (P3-4), the retinal afferents have stimulated the outgrowth of dendrites toward the zone of their heaviest concentration, opening up small spaces between layers. Additionally, cells or retinal afferents within the latter regions could be lost through lack of stimulation. Finally, between the first wave of cytoarchitectonic lamination and maturity, extraretinal afferents (especially from cortex) concentrate in the newly forming interlaminar spaces, stimulating a second wave of dendritic growth that further widens the interlaminar spaces. The end product of these interactions is the adult nucleus, which demonstrates afferent, cytological and cytoarchitectonic lamination. Last, the effects of bilateral eye enucleation at birth on the cytological and cytoarchitectonic characteristics of the nucleus are shown at the right. In this situation, no interlaminar spaces form because segregated retinal afferents are absent and the late-maturing non-retinal afferents from cortex (or perhaps other sources) distribute uniformly and "stimulate" uniform growth in the cells that survive (see also Casagrande and Brunso-Bechtold, 1985). 

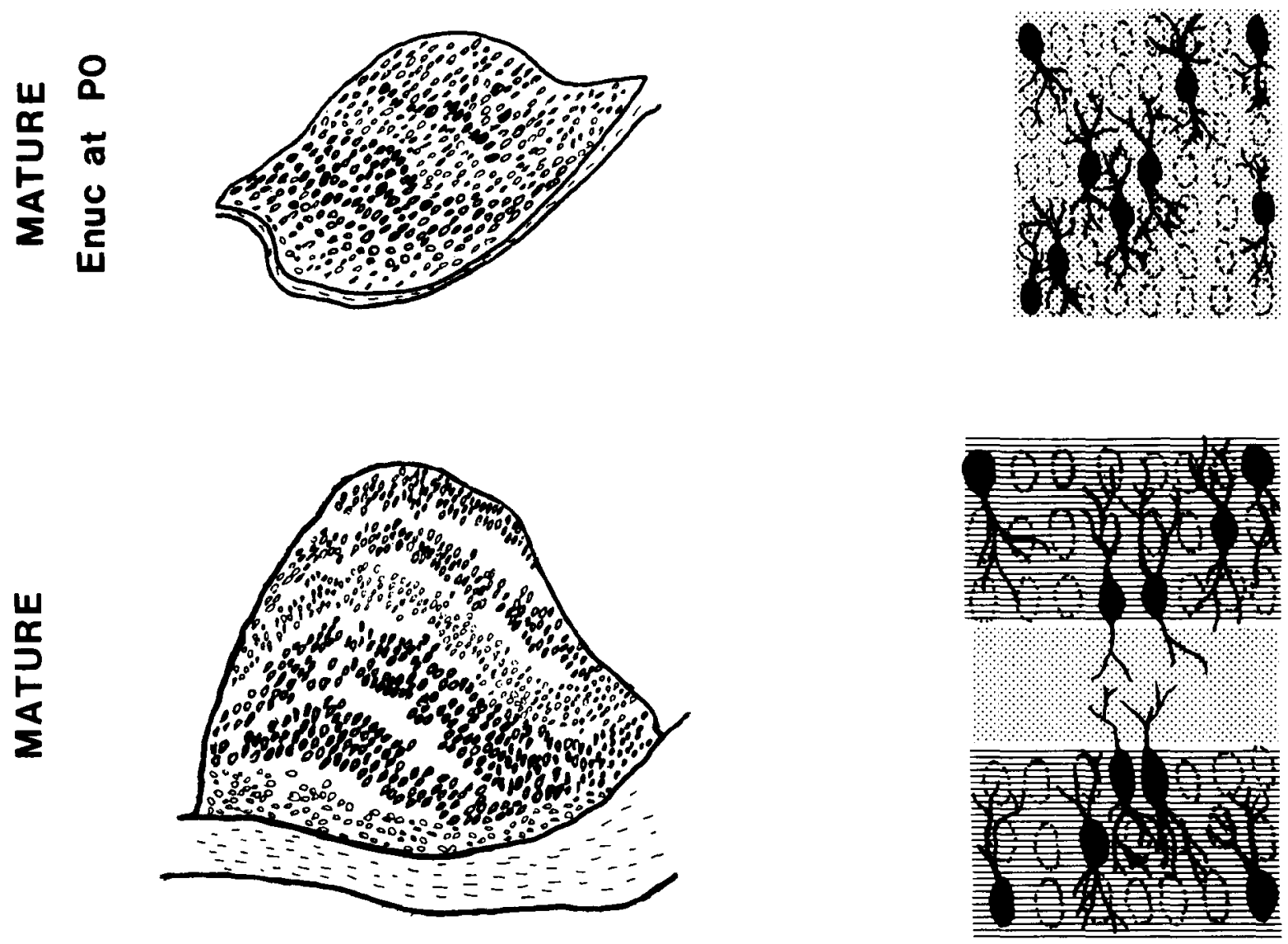

1
1
0
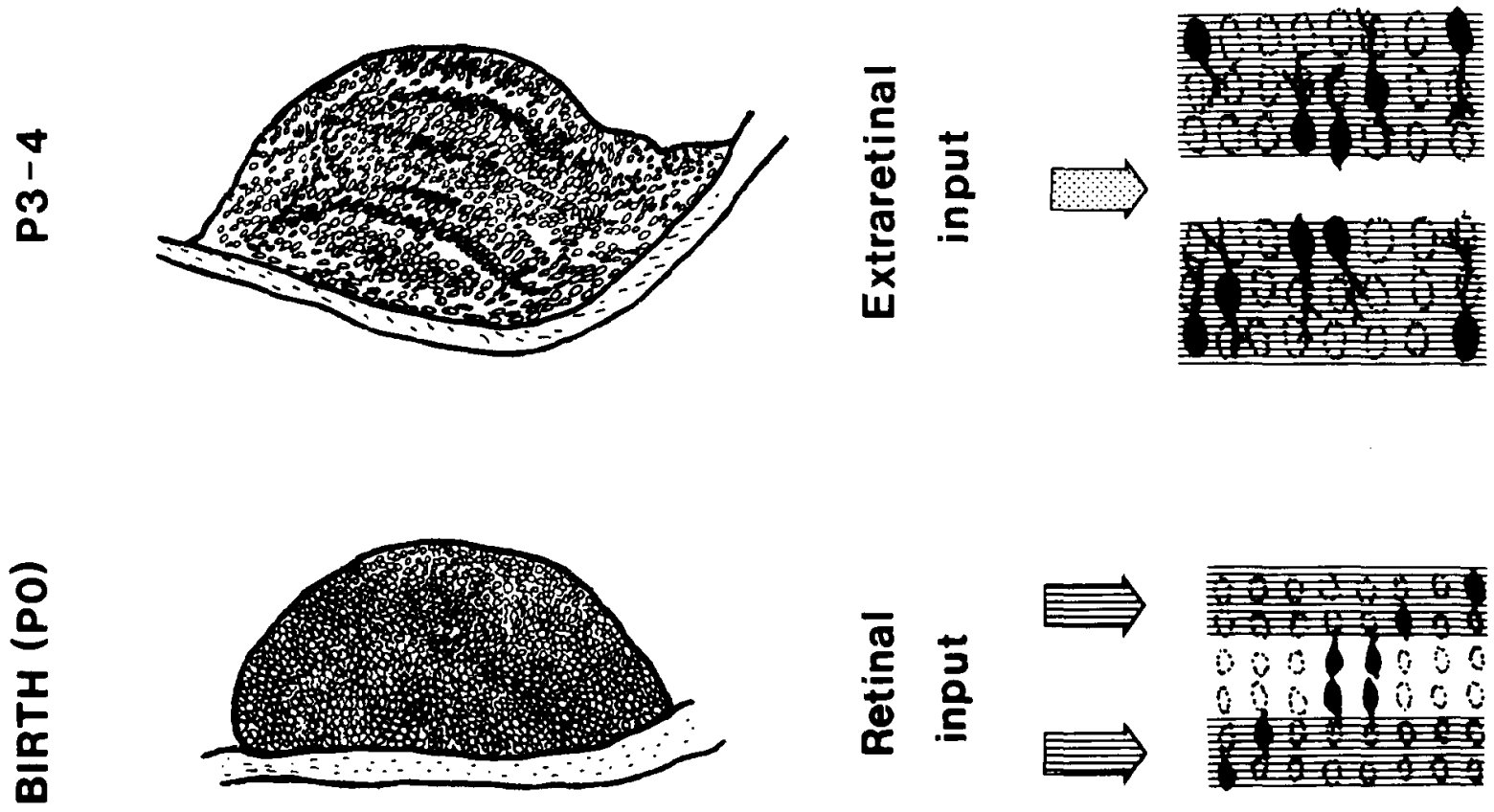
immature connections. Nevertheless, we should stress that, thus far, our evidence for such "sprouting" should be interpreted very cautiously, since it is based on only one case. Also, it is unclear why such sprouting was not apparent following bilateral TTX treatment, unless one postulates that the "normally" innervated LGN layers in the unilaterally treated animal somehow stimulate or attract the electrically silenced afferent axons.

\section{TTX treatment and enucleation}

Our results, combining TTX treatment in one eye with enucleation of the other eye, clearly show that the remaining, TTXblocked axons from one eye are capable of sustaining the portion of the nucleus to which they normally project, resulting in the production of a normal complement of LGN layers on each side of the brain (e.g., 2 layers on one side and 4 layers on the other side; see Fig. 14). As with tree shrews monocularly enucleated at birth and raised to adulthood, the LGNs of the combined monocularly enucleated and TTX-treated animals in this study exhibit a pattern of lamination suggesting that cells in both functionally matched sets of layers (i.e., layers 1 and 2 and layers 4 and 5) become innervated by the remaining eye, creating combined layers 1 and 2 or 4 and 5 (Casagrande and BrunsoBechtold, 1985; Casagrande and Condo, 1987). Nonetheless, the main point is that TTX treatment does not prevent retinogeniculate axons from providing their postsynaptic targets with important factors, factors that, when absent as a consequence of monocular eye removal, clearly place the deprived LGN cells at a severe competitive disadvantage.

\section{The role of activity in $L G N$ layer formation}

The mechanisms involved in LGN layer formation are still poorly understood. Evidence from the present study and the results of other studies demonstrate that neural activity plays an essential role in some, but not all, aspects of this process.

As mentioned earlier, preliminary results in kitten embroyos (Shatz and Stryker, 1986) indicate that blockade of a neural activity prevents segregation of retinal axons. Thus, spontaneous neural activity appears to be essential for one of the initial stcps in LGN layer formation.

Once retinogeniculate axons have segregated and rough retinotopic order is established, cell layers, defined by cell-free interlaminar spaces (i.e., cytoarchitectonic lamination), gradually begin to become visible. The present study has demonstrated that cytoarchitectonic lamination, unlike the process of segregation of retinogeniculate axons, develops in the absence of optic nerve action potential activity. Since we already know that interlaminar spaces in the LGN will not form in the absence of input from the retina (Brunso-Bechtold and Casagrande, 1981), the silent retinogeniculate axons must still be capable of providing the appropriate trophic factors to allow for the initiation of cellular lamination.

We have previously proposed (see Fig. 17) that interlaminar spaces could form through the release of such appropriate trophic factors, which, in turn, influence the differential adhesiveness of postsynaptic cells, such that cells of like type remain adherent, while those of unlike type separate. In addition, such trophic factors could influence the polarity of growth of LGN cell dendrites such that dendritic growth is polarized in the direction of the already segregated retinal afferents. In fact, we now have preliminary evidence in tree shrews that LGN cells at incipient interlaminar borders exhibit a strong tendency toward dendrite growth away from cellular borders (Casagrande et al., 1986; Lachica et al., 1987). In addition, the inception of interlaminar spaces coincides with the termination of nonretinal inputs within these spaces, which, we have argued, may also play a role in their formation (Brunso-Bechtold and Casagrande, 1983; Casagrande and Brunso-Bechtold, 1985). Finally, the release of retinogeniculate trophic factors could influence the viability of select populations of LGN cells helping to shape the laminar pattern. Thus far, however there is little evidence to support the latter possibility, since there are no reports of differential cell death in the LGN at the time interlaminar spaces form.

Results from the present study suggest that an appropriate level of neural activity within the retinogeniculate pathway is definitely important for the pace of some of these final developmental steps. In fact, the main result of silencing retinal ganglion cell action potential activity appears to be a dramatic slowing of the whole developmental process, such that retinogeniculate fibers, LGN cells, and interlaminar spaces all retain a very immature appearance.

At present, it is unclear how neural activity influences these final steps in LGN development. Certainly, we know that such activity continues to play an important role long after LGN layer formation has taken place and LGN cells are morphologically relatively mature. This is attested to by the many studies showing that both morphological and physiological changes can occur, based on visual experience in LGN cells in tree shrews and other species (Norton et al., 1977; Casagrande et al., 1978; see Sherman and Spear, 1982, for review). It is also unclear whether the activity-dependent mechanisms that allow retinogeniculate axons to influence LGN development are similar at different periods of development, or share features in common with the important trophic influence these axons clearly can provide their targets without such neural activity. A number of possibilities exist. One hypothesis is that neural activity influences the amount of a common trophic substance important in driving the maturation of both pre- and postsynaptic targets through intrinsic programs of development. According to this hypothesis, differences in the effect of blocking neural activity on LGN devclopment could simply be cxplaincd by the postulate that different phases of development require that different amounts of trophic factor be available in order to drive the system to the next developmental step. Some support for this view is provided by the recent experiments of Matthews (1985). Matthews demonstrated that blocking axoplasmic transport with intraocular injections of colchicine produced the same effect as did blocking activity with TTX eye injections (Cowan et al., 1984; Fawcett et al., 1984), namely, the normally transient expanded ipsilateral retinofugal projection within the superior colliculus was retained. Matthews (1985) also found that colchicine treatment retarded tectal synaptic differentiation. On the basis of these results, Matthews has suggested (see also Siebler and Schmidt, 1986) that similarities in the effects of blocking activity and blocking transport may be related to the common effect of blocking transport of specific glycoproteins seen following both types of manipulation (Edwards and Grafstein, 1983). In fact, several investigators have proposed that the transneural transfer of glycoproteins may be important in synchronizing developmental steps (see Specht and Grafstein, 1977).

Others have argued against the above hypothesis, suggesting that the small effects on axoplasmic transport that occur following TTX blockade of neural activity are not sufficient to account for effects on such events as eye-specific segregation of 
retinal axons (Reh and Constantine-Paton, 1985). If this is the case, then we would need to postulate separate mechanisms to account for effects of blockade of optic nerve activity on some, but not all, aspects of LGN laminar development, aspects that, nevertheless, require the presence and thus, presumably, the trophic influence of optic axons.

\section{References}

Brunso-Bechtold, J. K., and V. A. Casagrande (1981) Effects of bilateral enucleation on the development of layers in the dorsal lateral geniculate nucleus. Neuroscience 6: 2579-2586.

Brunso-Bechtold, J. K., and V. A. Casagrande (1982) Early postnatal development of laminar characteristics in the dorsal lateral geniculate nucleus of the tree shrew. J. Neurosci. 2: 589-597.

Brunso-Bechtold, J. K., and V. A. Casagrande (1983) Development of layers in the dorsal lateral geniculate nucleus in the tree shrew. In Contributions to Sensory Physiology, vol. 8, W. D. Neff, ed., pp. 4177, Academic, New York.

Brunso-Bechtold, J. K., and V. A. Casagrande (1985) Presence of retinogeniculate fibers is essential for initiating the formation of each interlaminar space in the lateral geniculate nucleus. Dev. Brain Res. 20: $123-126$.

Brunso-Bechtold, J. K., S. L. Florence, and V. A. Casagrande (1983) The role of retinogeniculate afferents in the development of connections between visual cortex and the dorsal lateral geniculate nucleus. Dev. Brain Res. 10: 33-39.

Casagrande, V. A. (1974) The laminar organization and connections of the lateral geniculate nucleus in the tree shrew (Tupaia glis). Anat. Rec. 178: 323.

Casagrande, V. A., and J. K. Brunso-Bechtold (1983) The relationship between afferent laminar development and cell layer formation in the lateral geniculate nucleus (LGN). Soc. Neurosci. Abstr. 9: 25.

Casagrande, V. A., and J. K. Brunso-Bechtold (1985) Critical events in lateral geniculate nucleus development. In Advances in Neurological and Behavioral Development, vol. 1, R. N. Aslin, ed., pp. 33-78, Ablex, Norwood, NJ.

Casagrande, V. A., and G. J. Condo (1988) Is binocular competition essential for layer formation in the lateral geniculate nucleus? Brain Behav. Fvol. (in press).

Casagrande, V. A., R. W. Guillery, and J. K. Harting (1978) Differential effects of monocular deprivation seen in different layers of the lateral geniculate nucleus. J. Comp. Neurol. 179: 469-485.

Casagrande, V. A., G. J. Condo, and L. A. Durden (1985) The effect of neural activity on the formation of geniculate cell layers. Soc. Neurosci. Abstr. 11: 805.

Casagrande, V. A., G. J. Condo, and E. A. Lachica (1986) Postnatal development of cytochrome oxidase staining in tree shrew lateral geniculate nucleus. Soc. Neurosci. Abstr. 12: 590.

Changeux, J. P., and A. Danchin (1976) Selective stabilization of developing synapses as a mechanism for the specification of neuronal networks. Nature 264: 705-712.

Constantine-Paton, M. (1982) The retinotectal hookup: The process of neural mapping. In Developmental Order: Its Origin and Regulation, S. Subtenly and P. B. Green, eds., Liss, New York.

Constantine-Paton, M., and M. I. Law (1978) Eye-specific termination bands in tecta of three-eyed frogs. Science 202: 639-641.

Conway, J. L., and P. H. Schiller (1983) Laminar organization of the tree shrew dorsal lateral geniculate nucleus. J. Neurophysiol. 50: 13301342.

Cowan, W. M., J. W. Fawcett, D. D. M. O'Leary, and B. B. Stanfield (1984) Regressive events in neurogenesis. Science 225: 1258-1265.

Dubin, M. W., L. A. Stark, and S. M. Archer (1986) A role for actionpotential activity in the development of neuronal connections in the kitten retinogeniculate pathway. J. Neurosci. 6: 1021-1036.

Edwards, D. L., and B. Grafstein (1983) Intraocular tetrodotoxin in goldfish hinders optic nerve regeneration. Brain Res. 269: 1-14.

Edwards, D. L., and B. Grafstein (1984) Intraocular injection of tetrodotoxin in goldfish decreases fast axonal transport of [ $\left.{ }^{3} \mathrm{H}\right]$ glucosamine-labeled materials in optic axons. Brain Res. 299: $190-194$.

Fawcett, J. W., and D. D. M. O'Leary (1985) The role of electrical activity in the formation of topographic maps in the nervous system. Trends Neurosci. 8: 201-206.
Fawcett, J. W., D. D. M. O'Leary, and W. M. Cowan (1984) Activity and the control of ganglion cell death in the rat retina. Proc. Natl. Acad. Sci. USA 81: 5589-5593.

Fitzpatrick, D., R. G. Carey, and I. T. Diamond (1980) The projection of the superior colliculus upon the lateral geniculate body in Tupaia glis and Galago senagalensis. Brain Res. 194: 538-542.

Guillery, R. W., A. S. Lamantia, J. A. Robson, and K. Huang (1985) The influence of retinal afferents upon the development of layers in the dorsal lateral geniculate nucleus of mustelids. J. Neurosci. 5: 13701379.

Holdefer, R. N., and T. T. Norton (1986) Laminar organization of receptive-field properties in the lateral geniculate nucleus of the tree shrew (Tupaia belangeri). Soc. Neurosci. Abstr. 12: 8.

Horton, J. C. (1981) Effect of blocking retinal ON-channels with aminophosphonobutyric acid (APB) on the cat visual system. Soc. Neurosci. Abstr. 7: 24.

Kalil, R. E., M. W. Dubin, G. L. Scott, and L. A. Stark (1983) Effects of retinal ganglion cell blockade on the morphological development of retinogeniculate synapses in the cat. Soc. Neurosci. Abstr. 9: 24.

Kalil, R. E., M. W. Dubin, G. Scott, and L. A. Stark (1986) Elimination of action potentials blocks the structural development of retino-geniculate synapses. Nature 323: 156-158

Knapp, A. G., and L. A. Mistler (1983) Response properties of cells in rabbit's lateral geniculate nucleus during reversible blockade of retinal ON-center channel. J. Neurophysiol. 50: 1236-1245.

Kuppermann, B. D., and T. Kasamatsu (1983) Changes in geniculate cell size following brief monocular blockade of retinal activity in kittens. Nature 306: 465-468.

Lachica, E. A., G. J. Condo, and V. A. Casagrande (1987) Development of cytochrome oxidase staining in the retina and lateral geniculate nucleus: A possible correlate of $\mathrm{ON}$ - and OFF-center channel maturation. Dev. Brain Res. 34: 297-302.

Matthews, M. A. (1985) Effects of neonatal intraocular colchicine on synaptogenesis and on the retention of the ipsilateral retinofugal projections within the superior colliculus. Exp. Brain Res. 60: 465-482.

McKanna, J. A., and V. A. Casagrande (1985) Computerized autoradiographic grain counting. In The Microcomputer in Cell and Neurobiology Research, chap. 16, R. Mize, ed., pp. 355-373, Elsevier, New York.

Mesulam, M. M. (1976) The blue reaction product in horseradish peroxidase neurohistochemistry: Incubation parameters and visibility. J. Histochem. Cytochem. 24: 1273-1280.

Norton, T. T., and M. Wong-Riley (1984) Cytochrome oxidase staining of $O N$ and OFF regions in the retina, LGN and striate cortex of tree shrew. Soc. Neurosci. Abstr. 10: 799.

Norton, T. T., V. A. Casagrande, and S. M. Sherman (1977) Loss of $Y$-cells in the lateral geniculate nucleus of monocularly deprived tree shrews. Science 197: 784-786.

Perry, V. H. (1982) The ganglion cell layer of the mammalian retina. In Progress in Retinal Research, N. Oshorne and G. Chader, eds., pp. 53-80, Pergamon, Oxford.

Rager, G., R. S. Nowakowski, S. Lausmann, M. Tanaka, and A. Schwaier (1980) The development of the retino-geniculo-cortical projection in the tree shrew, Tupaia belangeri. Soc. Neurosci. Abstr. 6: 662.

Rakic, P. (1986) Mechanism of ocular dominance segregation in the lateral geniculate nucleus: Competitive elimination hypothesis. Trends Neurosci. 9: 11-15.

Reh, T. A., and M. Constantine-Paton (1985) Eye-specific segregation requires neural activity in three-eyed Rana pipiens. J. Neurosci. 5 : $1132-1143$

Ricco, R. V., and M. A. Matthews (1983) Effects of tetrodotoxin upon axoplasmic transport in the developing visual system of the albino rat. Soc. Neurosci. Abstr. 9: 150.

Sandell, J. H., and P. H. Schiller (1982) The consequences of 2-amino4-phosphonobutyric acid (APB) eye injections in the monkey as determined by electrophysiolgoical and behavioral measures. Soc. Neurosci. Abstr. 8: 262.

Schiller, P. H. (1982a) Central connections of the retinal $O N$ and $O F F$ pathways. Nature 297: 580-583.

Schiller, P.H. (1982b) The effect of 2-amino-4-phosphonobutyric acid infusion into the retina on the responses of lateral geniculate and visual cortex neurons in the rhesus monkey. Invest. Ophthalmol. Vis. Sci. (Suppl.) 22: 11.

Schmidt, J. T. (1985) Formation of retinotopic connections: Selective 
stabilization by an activity-dependent mechanism. Cell. Mol. Neurobiol. 5: 65-84.

Schmidt, J. T., and D. L. Edwards (1983) Activity sharpens the map during the regeneration of the retinotectal projection in goldfish. Brain Res. 269: 29-39.

Schmidt, J. T., and S. B. Tieman (1985) Eye-specific segregation of optic afferents in mammals, fish and frogs: The role of activity. Cell. Mol. Neurobiol. 5: 5-34.

Shatz, C. J., and M. P. Stryker (1986) Tetrodotoxin infusion prevents the formation of eye-specific layers during prenatal development of the cat's retinogeniculate projection. Soc. Neurosci. Abstr. 12: 589.

Sherman, S. M., and P. D. Spear (1982) Organization of the visual pathways in normal and visually deprived cats. Physiol. Rev. 62: 738-855.

Siebler, M., and H. Schmidt (1986) Induction of the action potential in innervated slow muscle fibers of the frog: Effects of tetrodotoxin, vincristine and colchicine. Brain Res. 362: 299-307.

Slaughter, M. M., and R. F. Miller (1981) 2-Amino-4-phosphonobutyric acid: A new pharmacological tool for retina research. Science 211: 182-185.

Smith, E. L., III, D. A. Fox, and G. C. Duncan (1985) Refractive error changes in kitten eyes produced by chronic ON-channel blockade. Invest. Ophthalmol. Vis. Sci. (Suppl.) 26: 331.

Specht, S. C., and B. Grafstein (1977) Axonal transport and transneuronal transfer in mouse visual system following injection of $\left({ }^{3} \mathrm{H}\right)$ fucose into the eye. Exp. Neurol. 54: 352-368.
Sretavan, D. W., and C. J. Shatz (1984) Prenatal development of individual retinogeniculate axons during the period of segregation. Nature 308: 845-848.

Sretavan, D. W., and C. J. Shatz (1986) Prenatal development of retinal ganglion cell axons: Segregation into eye-specific layers within the cat's lateral geniculate nucleus. J. Neurosci. 6: 234-251.

Stryker, M. P., and W. A. Harris (1986) Binocular impulse blockade prevents formation of ocular dominance columns in the cat's visual cortex. J. Neurosci. 6: 2117-2133.

Sutton, J., and J. K. Brunso-Bechtold (1985) Effect of removal of retinal input on the development of the colliculogeniculate projection. Soc. Neurosci. Abstr. 11: 1015.

von der Marlsburg, C., and D. J. Willshaw (1981) Co-operativity and brain organization. Trends Neurosci. 4: 80-83.

Wong-Riley, M. (1979) Changes in the visual system of monocularly sutured or enucleated cats demonstrable with cytochrome oxidase histochemistry. Brain Res. 171: 11-28.

Wong-Riley, M., and E. Carroll (1984) Effect of impulse blockade on cytochrome oxidase activity in monkey visual system. Nature 307 : 262-264.

Wong-Riley, M. T. T., and D. A. Riley (1983) The effect of impulse blockade in the cytochrome oxidase activity in the cat visual system. Brain Res. 261: 185-193. 